\title{
Full title
}

Jasmonate biosynthesis arising from altered cell walls is prompted by turgor-driven mechanical compression and guides root hydrotropism

\section{Running title}

Enlarged root cortex trigger inner cells to make JA

\section{Authors}

Stefan Mielke ${ }^{1}$, Marlene Zimmer ${ }^{1}$, Mukesh Kumar Meena ${ }^{1}$, René Dreos ${ }^{2}$, Hagen Stellmach ${ }^{3}$, Bettina Hause $^{3}$, Cătălin Voiniciuc ${ }^{4}$ and Debora Gasperini ${ }^{1, *}$

\section{Affiliations:}

${ }^{1}$ Department of Molecular Signal Processing, Leibniz Institute of Plant Biochemistry, 06120 Halle (Saale), Germany.

${ }^{2}$ Center for Integrative Genomics, University of Lausanne, 1015 Lausanne, Switzerland. ${ }^{3}$ Department of Cell and Metabolic Biology, Leibniz Institute of Plant Biochemistry, 06120 Halle (Saale), Germany.

${ }^{4}$ Independent Junior Research Group - Designer Glycans, Leibniz Institute of Plant Biochemistry, 06120 Halle (Saale), Germany.

\section{Corresponding author}

* Debora Gasperini, Department of Molecular Signal Processing, Leibniz Institute of Plant Biochemistry, Weinberg 3, 06120 Halle (Saale), Germany, Tel: +49 (0) 34555821230

Email: debora.gasperini@ipb-halle.de 


\section{ABSTRACT}

Despite the vital roles of jasmonoyl-isoleucine (JA-Ile) in governing plant growth and environmental acclimation, it remains unclear what intracellular processes lead to its induction. Here, we provide compelling genetic evidence that mechanical and osmotic regulation of turgor pressure represents a key factor in eliciting JA-lle biosynthesis. After identifying cell wall mutant alleles in KORRIGAN1 (KOR1) with elevated JA-lle in seedling roots, we found that ectopic JA-lle resulted from cell nonautonomous signals deriving from enlarged cortex cells compressing inner tissues and stimulating JA-lle production. Restoring cortex cell size by cell-type-specific KOR1 complementation, by isolating a genetic kor1 suppressor, and by lowering turgor pressure with hyperosmotic treatments, abolished JA-Ile signalling. Strikingly, heightened JA-lle levels guided kor1 roots towards greater water availability, uncovering a previously unrecognized JA-lle function in root hydrotropism. Collectively, these findings enhance our understanding of JA-lle biosynthesis initiation, and reveal a novel role of JA-lle in orchestrating environmental resilience.

\section{INTRODUCTION}

In higher plants, the phytohormone (+)-7-iso-jasmonoyl-L-isoleucine (JA-Ile) is a decisive coordinator of growth and stress responses (1). Induced JA-lle signalling secures a successful execution of reproductive development; regulates the acclimation to unfavourable conditions such as drought, salt, cold, elevated ozone and ultra violet light; and is essential to protect plants against insect herbivory, necrotrophic pathogens and RNA viruses (1-3). It is estimated that insect herbivory alone impacts $>20 \%$ of global net plant productivity, and plants unable to synthesize JA-lle, such as the allene oxide synthase (aos) mutant of the model plant Arabidopsis thaliana, are unprotected against chewing insects $(4,5)$. Rising hormone levels elicit nuclear JA-lle perception and signalling, leading to the increased expression of JA-Ile marker genes, such as JASMONATE-ZIM DOMAIN 10 (JAZ10), and upregulation of JA-lle induced defense transcripts such as VEGETATIVE STORAGE PROTEIN 2 (VSP2) and PLANT DEFENSIN 1.2 (PDF1.2) (1, 6, 7). As mounting defense responses are accompanied by reduced vegetative growth, JA-lle levels are tightly regulated and normally induced only when required $(1,8)$. Despite the broad and critical functions of the jasmonate (JA) pathway in plant growth and environmental acclimation, the intracellular events triggering JA-lle production remain poorly understood $(1,9,10)$.

Several molecular elicitors of JA-Ile biosynthesis have been proposed, including herbivore-, microbe-, and damage-associated molecular patterns generated during environmental stresses. These elicitors would be recognized by putative pattern recognition receptors at the cell surface that transduce the 
signal intracellularly and initiate de novo JA-lle synthesis, reviewed in $(9,11)$. However, conclusive genetic evidence for ligand-based induction of JA-Ile biosynthesis is still missing. Mechanical wounding is another potent activator of JA-lle biosynthesis and is widely used to stimulate endogenous hormone production $(6,10)$. In parallel to necrotroph and herbivorous insect attacks, mechanical wounding causes the disruption of cell walls which surround each plant cell and serve as the immediate contact surface with the extracellular environment. Plant cell walls are elaborated structural networks consisting predominantly of complex polysaccharides (cellulose, hemicelluloses and pectins), a lower amount of proteins, and other soluble and phenolic material (12). Notably, chemical or genetic inhibition of cellulose biosynthesis by isoxaben application or by mutations in CELLULOSE SYNTHASE (CesA) genes leads to increased JA-lle levels, eg. $(11,13,14)$. Consistently, mutations in KORRIGAN1 (KOR1), a CesA-interacting membrane protein with endo-1,4-beta-Dglucanase activity involved in cellulose biosynthesis, exhibits a slight basal increase in the JA-Ile precursor JA (15-19). Nevertheless, it remains unclear how cellulose deficiency stimulates JA-Ile production and in which plant tissues the hormone accumulates.

Plant cells have a high intracellular turgor pressure deriving from a gradient in osmotic potential across the plasma membrane, which is counterbalanced by their cell walls with cellulose microfibrils serving as a load-bearing component (12). If osmotic conditions change, plant cells may experience mechanical stress and even deform their shape depending on the extent of the stimulus, their geometry and the properties of their cell walls. Hence, they regulate water and ionic fluxes across the plasma membrane and remodel their cell wall until a new osmotic equilibrium is reached and turgor pressure is re-established $(20,21)$. Cell walls with compromised cellulose microfibrils may be inefficient at counteracting the high intracellular turgor pressure, resulting in enlarged cells. Cell swelling is in fact a typical feature observed in cellulose-deficient mutants or isoxaben-treated plants $(22,23)$. Mechanical stress arising from turgor pressure changes might be therefore involved in activating JA-mediated stress responses (24). Interestingly, co-treatments of liquid-grown seedlings with isoxaben and sorbitol serving as osmotica nullified the increased JA levels induced by isoxaben alone $(23,25)$, linking osmoregulation to the JA pathway.

Here, we investigated how cues derived from perturbed cellulose biosynthesis integrate with JA-lle production. First, we identified kor1 alleles with increased JA-Ile levels in cell-type-specific contexts of the primary root. While increased JA-lle signalling in kor1-4 was confined to inner root tissues, restoring KOR1 function in adjacent cortex cells complemented the JA phenotype. Transversal sections revealed a pronounced enlargement of cortex cells, likely exerting mechanical pressure on 
physically constrained inner tissues. In fact, reducing cortex cell size by genetic and chemical means fully abolished expanded kor1 cortex cells and restored JA-Ile signalling, indicating that jasmonate biosynthesis is triggered by turgor-driven mechanical compression. Notably, constitutive JA-Ile signalling did not impact kor1 growth rate nor its defense responses, but was crucial to guide root growth towards greater water availability.

\section{RESULTS}

\section{Cellulose-deficient kor1 mutants have increased root Jasmonate levels}

In a forward genetic screen aimed at identifying negative regulators of JA signalling (6), we isolated two alleles of KOR1, kor1-4 and kor1-5, exhibiting ectopic expression of the JA-responsive reporter JAZ10p:GUSPlus (JGP). Basal JGP expression in the wild type (WT) as well as in JA-deficient aos plants is very weak, whereas kor1 alleles showed JGP activation in the primary root (Fig. 1A, Fig. S1A). Allelism tests with a T-DNA insertion kor1-6 mutant in the JGP background and complementation by transformation with untagged (KOR1p:KOR1) and N-terminal CITRINE (CIT)-tagged KOR1 (KOR1p:CITKOR1) constructs, both restoring the kor1 short root length, confirmed the causative mutations of the observed JGP phenotype (Fig. 1A and B). EMS kor1 alleles harboured single amino acid exchanges within the extracellular GLYCOSYL HYDROLASE 9 (GH9) domain of KOR1 (L573F in kor1-4 and P172L in kor1-5), while kor1-6 resulted in lower KOR1 transcript levels (Fig. S1B and C). Among the three kor1 alleles, kor1-5 showed the most severe JA and root elongation phenotypes and kor1-6 the mildest (Fig.1A and C, Fig. S1A and D), implying that all mutants still retain partial KOR1 function. This is in line with previous reports proposing that full KOR1 knock-outs are lethal (16). Given the milder growth defects and robust JA phenotype of kor1-4, we employed this allele for further analyses.

Despite the broad KOR1 expression domain and stunted shoot growth phenotypes of characterized mutants (26), constitutive JGP activation was not detected in aerial tissues of kor1 alleles (Fig. 1A, Fig. S1A). Shoot wounding and exogenous MeJA treatment promptly induced JGP reporter expression across kor1 tissues, validating the root specificity of basal JGP activity (Fig. 1A, Fig. S1A). Quantitative analysis of JAZ1O transcripts further supported the specific activation of JA signalling in roots but not shoots of all three kor1 alleles (Fig. 1C). This phenotype was dependent on JA-Ile production, as increased reporter activity and JAZ10 transcript levels were abolished in JA-deficient kor1 aos double mutants (Fig.1A, Fig. S1A, E and F). In addition to JAZ10, kor1-4 exhibited increased root expression of JA marker transcripts JASMONATE OXYGENASE 3 (JOX3) and JAZ3 in a JAdependent manner, as well as increased levels of the bioactive JA-lle conjugate (Fig. 1D and E, Fig. 
S1G). Collectively, our data indicate that KOR1 is a negative regulator of root JA-lle biosynthesis.

Hence, kor1 mutants represent valuable genetic tools to study how are cell-wall derived signals integrated with intracellular hormone production, and uncover JA-lle functions in moderating root responses to cellulose-deficiency.

A

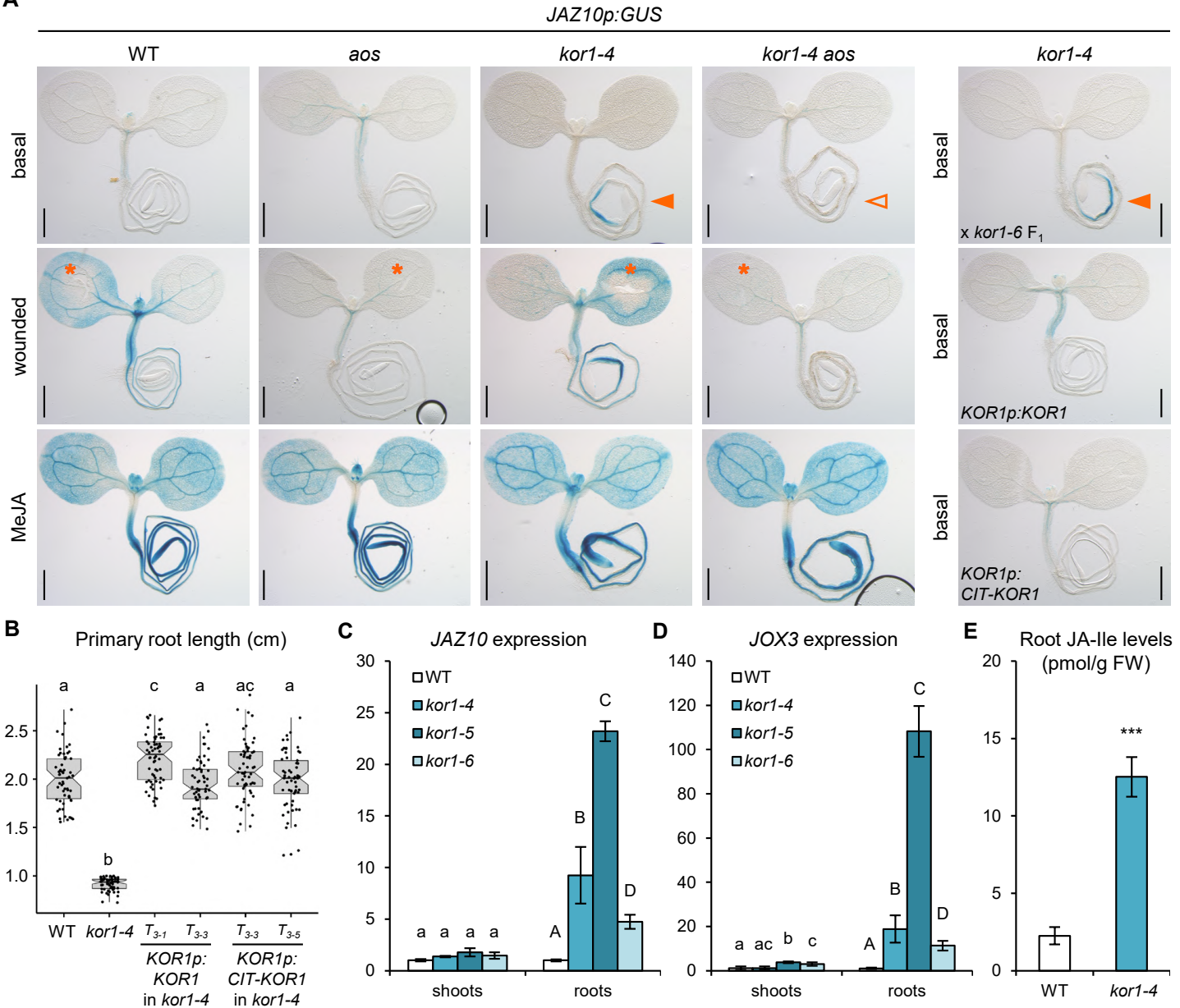

Fig. 1 kor1 mutant roots display increased jasmonate levels and signalling.

(A) Representative JAZ10p:GUS reporter activity in 5-do seedlings of WT, aos, kor1-4, kor1-4 aos at basal conditions, $2 \mathrm{~h}$ after cotyledon wounding (orange asterisks), $2 \mathrm{~h}$ after $25 \mu \mathrm{M}$ MeJA treatment, and in kor1-4 x kor1-6 $\mathrm{F}_{1}$ (allelism test), kor1-4 complemented with KOR1p:KOR1 and KOR1p:CITKOR1 lines. Note the increased JAZ1Op:GUS reporter activity in kor1-4 and allelism test (orange arrowheads), and its absence from kor1-4 aos (empty arrowhead). Scale bars, $0.5 \mathrm{~mm}$. (B) Primary root length box plot summary in 7-do WT, kor1-4, and two independent $\mathrm{T}_{3}$ lines for each complementing KOR1p:KOR1 and KOR1p:CIT-KOR1 construct. Both constructs restored the kor1-4 short root phenotype to WT length. Medians are represented inside the boxes by solid lines, circles depict individual measurements $(n=59-61)$. (C and D) Quantitative RT-PCR (qRT-PCR) of basal (C) JAZ10 and (D) JOX3 expression in shoots and roots of WT and 3 kor1 mutant alleles. JAZ1O and JOX3 transcript levels were normalized to those of $U B C 21$. Bars represent the means of 3 biological replicates $( \pm S D$ ), each containing a pool of $\sim 60$ organs from 5 -do seedlings. (E) Absolute JA-lle content in WT and kor1-4 roots. Bars represent the means of three biological replicates ( $\pm S D$ ), each containing a pool of $\sim 600$ roots from 5 -do seedlings. Letters and asterisks denote statistically significant differences among samples as determined by ANOVA followed by Tukey's HSD test $(\mathrm{P}<$ $0.05)$ in $(B-D)$, and by Student's t-test $(P=0.0005)$ in $(E)$. 

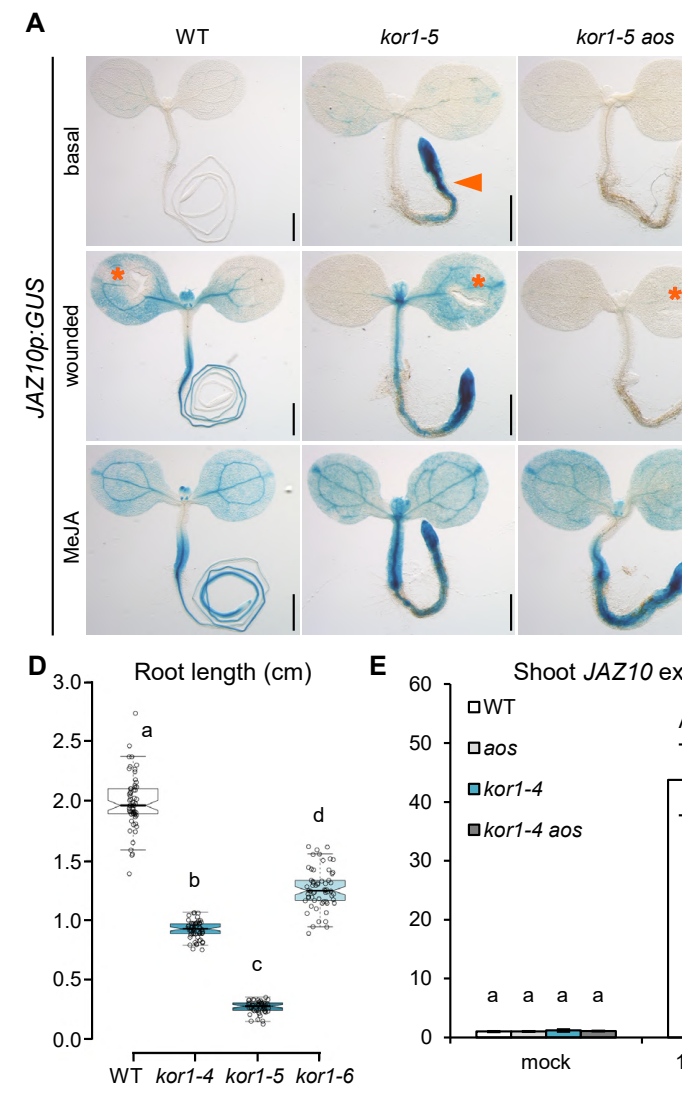

kor1-6

B
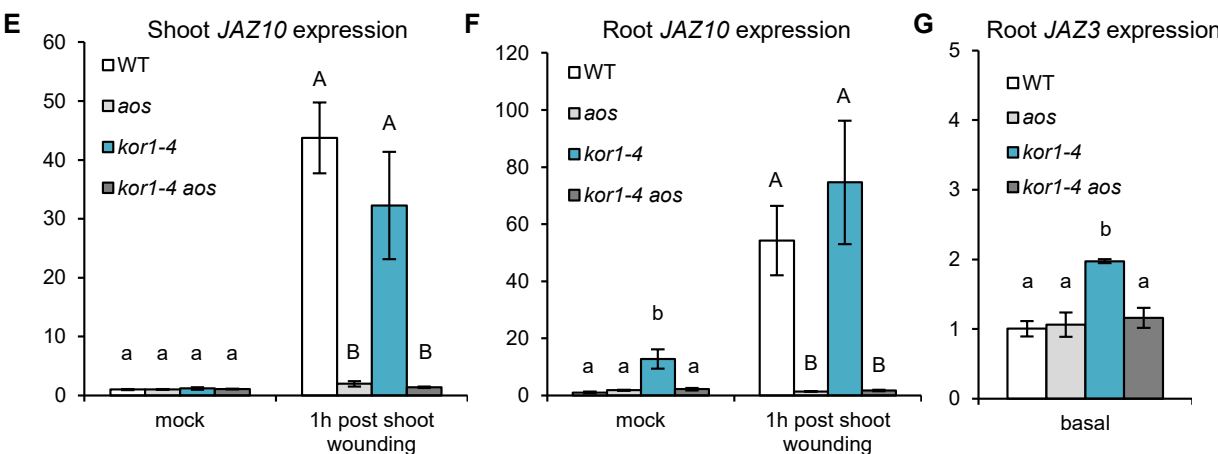

Fig. S1 Characterization of JA signalling in kor1 alleles.

(A) Representative JAZ10p:GUS reporter activity in 5-do seedlings of indicated genotypes basally, $2 \mathrm{~h}$ after cotyledon wounding (orange asterisks), and $2 \mathrm{~h}$ after $25 \mu \mathrm{M}$ MeJA treatment. Note the increased JAZ1Op:GUS reporter activity in roots of kor1-5 and kor1-6 mutants (orange arrowheads), and its absence from kor1-5 aos roots (empty arrowhead). Scale bars, $0.5 \mathrm{~mm}$. (B) Schematic representation of KOR1 gene structure describing the 3 kor 1 alleles used in this study, and primers (blue arrowheads) used to assess KOR1 expression by RT-PCR in (C). Black boxes depict exons and lines introns. (C) Semi-quantitative RT-PCR of KOR1 transcripts in WT and 3 kor 1 alleles. Note that kor1-4 and kor1-5 are point mutants while the T-DNA insertion in kor1-6 resulted in a mild reduction in KOR1 transcript abundance. (D) Primary root length box plot summary of 7-do seedlings in indicated genotypes. Medians are represented inside the boxes by solid lines, circles depict individual measurements $(\mathrm{n}=51-61)$. ( $\mathrm{E}$ and $\mathrm{F})$ qRT-PCR of JAZ10 expression basally and $1 \mathrm{~h}$ after shoot wounding in (E) aerial organs and (F) roots of WT, aos, kor1-4 and kor1-4 aos. (G) qRT-PCR of JAZ3 basal levels in roots of WT, aos, kor1-4 and kor1-4 aos. Transcript levels in (E) to (G) were normalized to those of $U B C 21$ and displayed relative to WT controls. Bars in (E) to (G) represent the means of three biological replicates $( \pm S D)$, each containing a pool of organs from $\sim 605$-do seedlings. Letters denote statistically significant differences among samples as determined by ANOVA followed by Tukey's HSD test $(P<0.05)$ in (D) to (G).

\section{Cortex-specific KOR1 expression complements ectopic JA signalling in kor1 endodermis and} pericycle

To map the precise tissues and cell types displaying ectopic JA signalling in kor1-4 roots, we used a transcriptional reporter expressing three VENUS (3xVEN) fluorescent proteins fused $\mathrm{N}$-terminally to a NUCLEAR LOCALIZATION SIGNAL (NLS) under the control of JAZ1Op (JAZ10p:NLS-3xVEN) (27). 
Similar to JGP, JAZ10p:NLS-3xVEN expression was not detectable at basal conditions but increased significantly after MeJA treatment in both WT and aos roots, while mechanical wounding triggered JAZ10p:NLS-3xVEN induction in the WT but not in aos (Fig.2A, Fig. S2). In contrast to the WT, kor1-4 exhibited constitutive JAZ10p:NLS-3xVEN expression predominantly in the early differentiation zone of the primary root, mostly confined to endodermal and pericycle cells (Fig. 2A to C). The extent of activated JA signalling was quantified by evaluating the presence of JAZ1Op:NLS-3xVEN along longitudinal cell files, and displaying the resulting frequency in a heatmap for each cell layer (Fig. 2D). Weak reporter activation coincided with the onset of cell elongation and proceeded longitudinally into the early differentiation zone for approximately 30 cells before ceasing. While only $<10 \%$ of kor1-4 roots showed sporadic JAZ1Op:NLS-3xVEN expression in a few epidermal or cortex cells, the majority of individuals displayed consistent JA signalling in a stretch of 10-15 endodermal and pericycle cells in the root early differentiation zone (Fig. 2D).

Because JA-lle precursors can relocate across tissues $(28,29)$, increased JA signalling in different cell types could result from cell autonomous or non-autonomous signals. To discriminate between these two possibilities and identify the source tissue responsible for increased JA-lle biosynthesis, we drove the expression of a functional CIT-KOR1 fusion protein under the control of cell-type-specific promoters and evaluated its capacity to complement the ectopic JGP expression in kor1-4. As expected, when driven by its endogenous promoter (KOR1p), CIT-KOR1 expression was detectable across the entire root, and respective cell-type-specific promoters resulted in CIT-KOR1 expression at the intended locations: IRON-REGULATED TRANSPORTER 1 (IRT1p) in the epidermis, PLASTID ENDOPEPTIDASE (PEPp) in the cortex, SCARECROW (SCRp) in the endodermis, and WOODEN LEG 1 $(W O L 1 p)$ in the stele which includes the pericycle (Fig. S3A to D) $(26,30)$. Intriguingly, the ectopic JGP expression in kor1-4 was complemented only when expressing CIT-KOR1 in the cortex (PEPp:CITKOR1), but not in the epidermis (IRT1p:CIT-KOR1), nor in endodermal (SCRp:CIT-KOR1) or pericycle (WOLp:CIT-KOR1) cells which exhibited high JA signalling (Fig. 2E). Furthermore, the short root kor14 phenotype was ameliorated by expressing CIT-KOR1 in the cortex, while it remained unchanged when expressing the construct in the epidermis, and it was even slightly exacerbated by cell-typespecific expression in the endodermis or pericycle (Fig. S3E). These results suggest that maintaining KOR1 functionality in the cortex is critical for impeding the activation of JA-Ile production in adjacent endodermal and pericycle cells. 

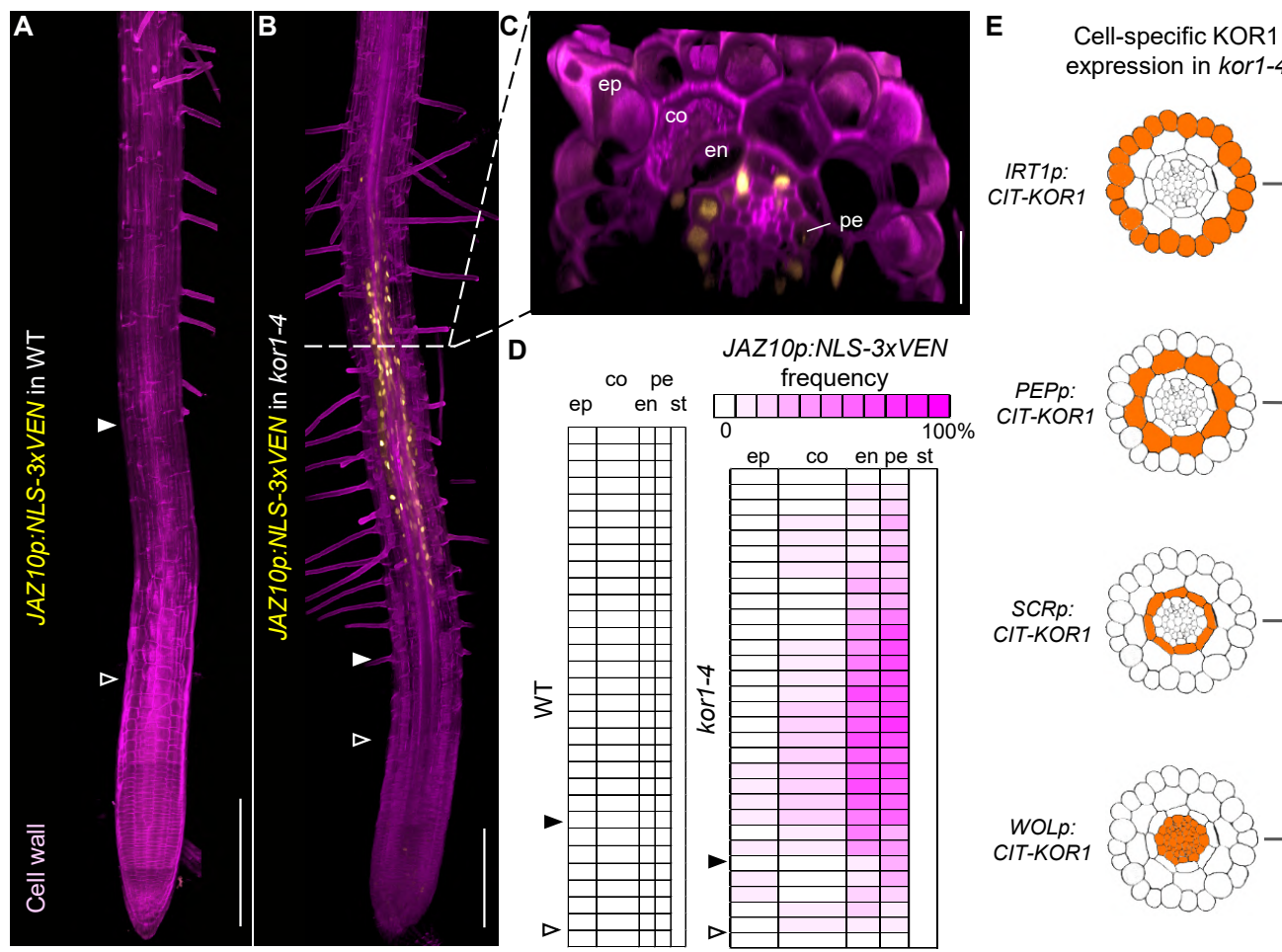

JAZ10p:GUS

JAZ10p:NLS-3xVEN
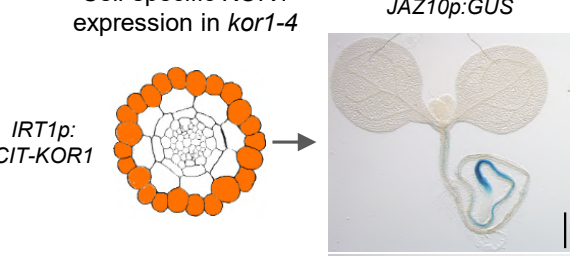

Fig. 2 Cortex-specific expression of CIT-KOR1 complements increased JA signalling in endodermis and pericycle of kor1-4.

(A to C) JAZ10p:NLS-3xVEN expression in 5-do (A) WT and (B and C) kor1-4 roots cleared with ClearSee, counterstained with the cellulose dye Direct Red 23 and visualized as 3D Z-stacks. (C) Orthogonal view from the epidermis to the vascular cylinder of kor1-4. The onset of elongation is indicated by empty arrowheads (first elongated cortex cell), and that of differentiation by filled arrowheads (appearance of root hairs). ep, epidermis; co, cortex; en, endodermis; pe, pericycle. (D) Heatmap of JAZ1Op:NLS-3xVEN frequency in individual cells from WT and kor1-4 primary roots $(\mathrm{n}=$ 21). Presence or absence of the reporter was evaluated from the onset of elongation in individual cells along consecutive longitudinal files for each tissue layer. Reporter expression was not observed in the WT nor in kor1-4 vascular tissues of the stele (st). (E) Cell-specific complementation of JAZ1Op:GUS activity in kor1-4 by expressing CIT-KOR1 in either epidermis (IRT1p:CIT-KOR1), cortex (PEPp:CIT-KOR1), endodermis (SCRp:CIT-KOR1) or pericycle and stele (WOLp:CIT-KOR1). Note that cortex-expressed CIT-KOR1 complements JAZ1Op:GUS activity in kor1-4, as indicated by lack of the JAZ10p:GUS reporter in representative images from $\mathrm{T}_{3}$ lines (empty orange arrowhead). Scale bars, $200 \mu \mathrm{m}(\mathrm{A}$ and $\mathrm{B}), 30 \mu \mathrm{m}(\mathrm{C})$, and $0.5 \mathrm{~mm}(\mathrm{E})$. 


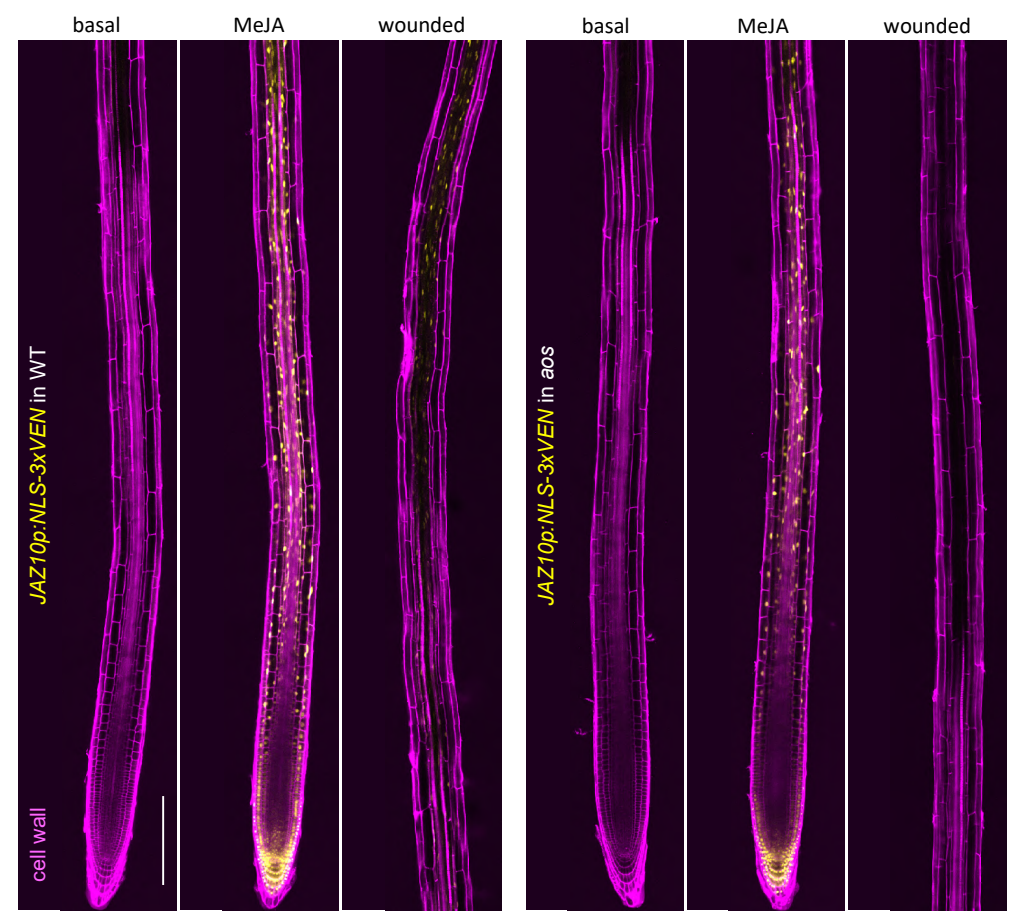

Fig. S2 Validation of the JAZ10p:NLS-3xVEN reporter.

JAZ10p:NLS-3XVEN reporter expression in WT and aos 5-do roots at mock (basal) conditions, $2 \mathrm{~h}$ after $10 \mu \mathrm{M}$ MeJA treatment and $2 \mathrm{~h}$ after cotyledon wounding $(\mathrm{n}=10)$. Cell wall pectins were counterstained with Propidium lodide. Note that the reporter was activated broadly in WT and aos after MeJA treatment while wound-induced activation did not reach the root meristem and was constrained to vascular tissues. Scale bar, $200 \mu \mathrm{m}$.
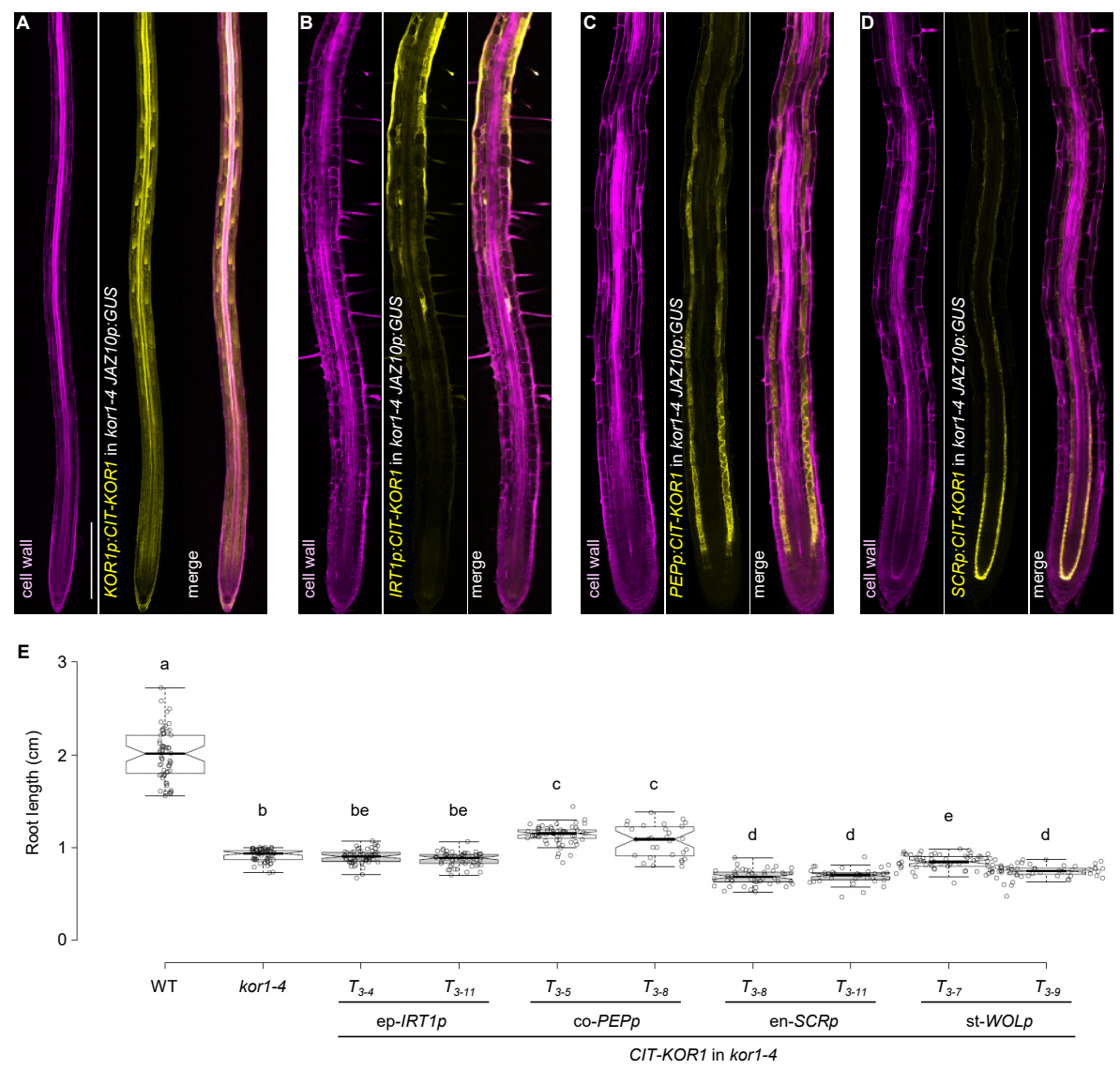
Fig. S3 Cell-type specific CIT-KOR1 expression in kor1-4 and relative root lengths.

(A to D) CIT-KOR1 expression in 5-do kor1-4 JGP seedling roots under the control of (A) the native KOR1 promoter (KOR1p:CIT-KOR1), (B) the epidermal IRT1 promoter (ep-IRT1p, IRT1p:CIT-KOR1), (C) the cortex PEP promoter (Co-PEPp, PEPp:CIT-KOR1), (D) the endodermal SCR promoter (en-SCRp, SCRp:CIT-KOR1). For WOLp:CIT-KOR1, we have observed extremely weak CIT fluorescence in pericycle and stele only at higher detector gains. Samples were cleared in ClearSee and counterstained with the cellulose dye Direct Red 23. Scale bar, $200 \mu \mathrm{m}$. (E) Primary root length box plot summary of 7-do seedlings in indicated genotypes. Medians are represented inside the boxes by solid lines, circles depict individual measurements $(n=30-60)$, and letters denote statistically significant differences among samples as determined by ANOVA followed by Tukey's HSD test $(\mathrm{P}<$ $0.05)$. Note that WT and kor1-4 values are in common with Fig. 1B.

\section{Mutations in ESMD1 suppress elevated JA signalling levels in kor1}

To identify genetic regulators of JA-lle production in kor1, we performed an EMS suppressor screen in kor1-4 JGP for lack of JA reporter activation. Mapping-by-whole-genome-sequencing of bulk segregants isolated an allele in ESMERALDA1 (ESMD1), which we named esmd1-3. The esmd1-3 allele abolished the constitutive JGP expression and high JAZ10 levels in kor1-4 roots while retaining the capacity to induce JAZ10 transcripts after wounding (Fig. 3A and B, Fig. S4A to C). An ESMD1mTURQUOISE2 $(\mathrm{mT})$ fusion protein expressed under the ESMD1p native promoter restored the ectopic JA signalling in kor1-4 esmd1-3 (Fig. 3A, Fig. S4A). Consistently, introgressing another esmd11 EMS allele (31) into kor1-4 partially suppressed the elevated JAZ10 levels in kor1-4 (Fig. 3B), confirming the suppressor's identity and causative amino acid R373C substitution in esmd1-3 (Fig. S4D). As we could not retrieve homozygous mutants from a segregating $F_{2}$ esmd1 population (GABI_216D03), and esmd1-1 was a weaker JA suppressor than esmd1-3, it is likely that full loss of ESMD1 function leads to lethality and that both alleles used herein are partial loss-of-function. ESMD1 is a member of the plant-specific glycosyltransferase GT106 family, with putative $O$ fucosyltransferase activity involved in regulating pectin homeostasis $(31,32)$. Specifically, esmd1 was identified as a suppressor of the stunted growth phenotypes of mutants deficient in the major pectin constituent homogalacturonan (HG) (31). Because esmd1 restored the cell-to-cell adhesion defects without complementing the low HG content of pectin mutants quasimodo1 (qua1, a putative galacturonosyltransferase of the glycosyltransferase GT8 family) and quasimodo2 (qua2, a methyltransferase) (31, 33-35), we wondered if in addition to suppressing JA signalling, esmd1-3 was able to revert other kor1-4 phenotypes.

Contrary to ameliorating qua1 and qua2 growth phenotypes, esmd1-3 did not restore the short root length nor impacted cellulose content in both shoots and roots of kor1-4 seedlings (Fig. 3C and D, Fig. S4E). Monosaccharide composition analysis did not reveal major changes in hemicellulose nor pectin constituents between WT and kor1-4 in both shoots and roots (Fig. 3E, Fig. S4F). 
Consequently, and in line with previous reports (31), we expected similar neutral and acidic sugar profiles from cell walls of genotypes with esmd1-3. Nonetheless, a conspicuous reduction in rhamnose abundance was detected in esmd1-3 genotypes with respect to the WT, 32-38\% in roots and $15 \%$ in shoots (Fig. 3E, Fig. S4F). Since cellulose-deficiency often leads to ectopic lignification (36), we next evaluated lignin deposition in our mutant set. Phloroglucinol staining revealed patches of lignified cells across the primary root of kor1-4 that were absent in WT and aos plants (Fig. 3F, Fig. S4G). The ectopic lignification in kor1-4 was not a consequence of elevated JA levels as kor1-4 aos still exhibited lignified cells, and was entirely abolished in kor1-4 esmd1-3. Overall, the cell wall analysis indicated an elaborated compensatory network in the kor1 esmd1 double mutant, in which some phenotypes were epistatic to kor1 (short root length, cellulose deficiency) and others to esmd1 (lack of increased JA signalling, reduced rhamnose abundance, lack of ectopic lignification). The results therefore suggest that increased JA signalling in kor1 may be due to indirect consequences of cellulose deficiency, rather than to altered levels of a specific cell wall component.

To gain further insights on how ESMD1 may influence JA responses in kor1, we concentrated on its localization in the primary root. Although an ESMD1-GFP fusion protein was expressed in the Golgi when transiently overexpressed in leaf epidermal cells of Nicotiana benthamiana (31), we were unable to visualize the functional ESMD1p:ESMD1-mT construct nor ESMDp:ESMD1-CIT in WT Arabidopsis roots, suggesting ESMD1 levels are very low and/or tightly regulated. Instead, ESMD1p:NLS-3xVEN expression was mapped to older parts of the root as expected (31), and to the epidermis, cortex and endodermis of the early differentiation zone (Fig. $3 \mathrm{G}$ to I). 


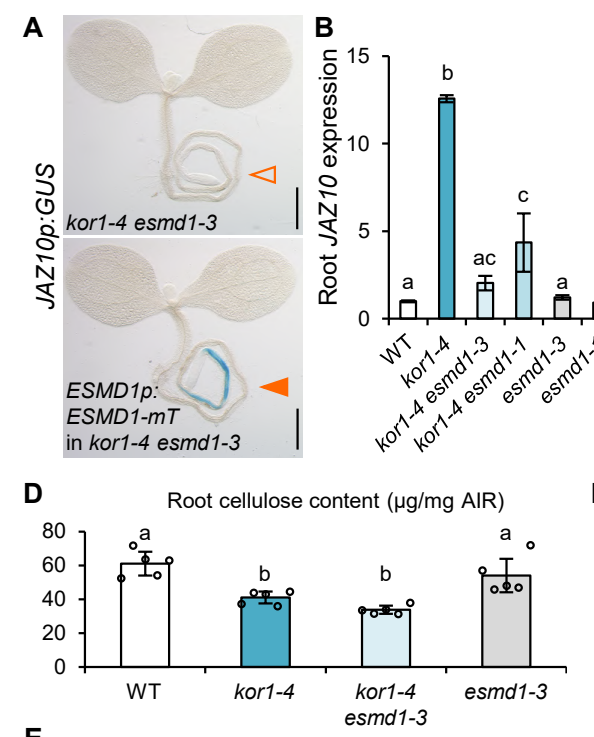

$E$

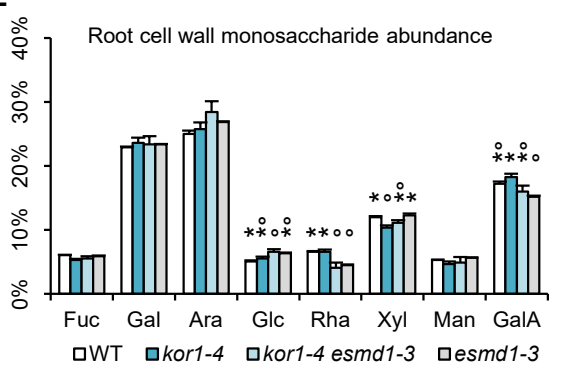

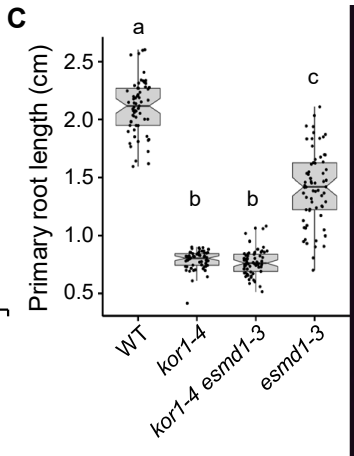

$\mathbf{F}$
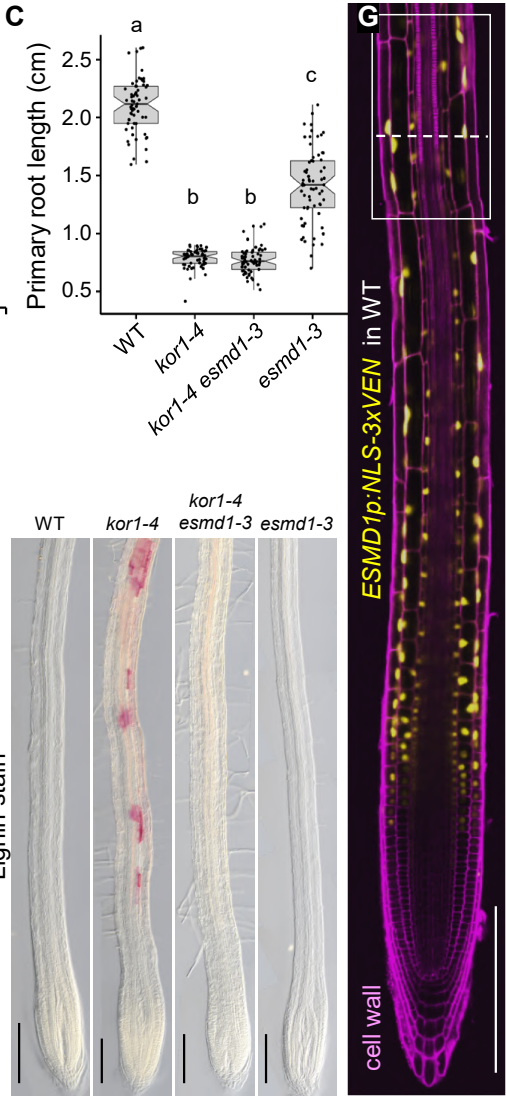

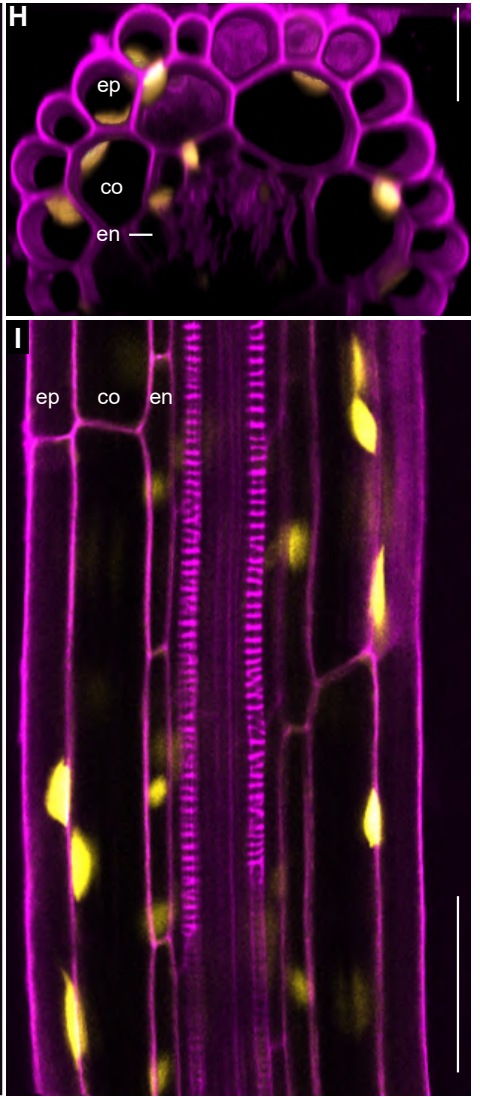

Fig. 3 ESMD1 is a positive regulator of ectopic JA-lle production in kor1.

(A) JAZ10p:GUS expression in kor1-4 esmd1-3 and its ESMD1p:ESMD1-mTurquoise $(m T)$ complementation line. Note the lack of JAZ10p:GUS reporter activity in roots of kor1-4 esmd1-3 (empty arrowhead), and its presence in the complemented line (orange arrowhead). (B) qRT-PCR of basal JAZ10 expression in roots of indicated genotypes. JAZ10 transcript levels were normalized to those of $U B C 21$. Bars represent the means of three biological replicates ( $\pm S D$ ), each containing a pool of 60 organs from 5-do seedlings. (C) Primary root length box plot summary in 7-do WT, kor14, kor1-4 esmd1-3 and esmd1-3 seedlings. Medians are represented inside the boxes by solid lines, circles depict individual measurements $(n=58-66)$. (D) Crystalline cellulose content from alcohol insoluble residue (AIR) extracted from roots of indicated genotypes. Bars represent means of five biological replicates depicted as circles $( \pm S D)$, each consisting of pools from $\sim 300$ roots from 12 -do seedlings. (E) Cell wall monosaccharide composition analysis from AIR extracted from roots of indicated genotypes. Bars represent the means of three biological replicates $( \pm S D)$, each containing a pool of $\sim 300$ roots from 12-do seedlings. Fuc, fucose; Gal, galactose; Ara, arabinose; Glc, glucose; Rha, rhamnose; Xyl, xylose; Man, mannose; GalA, Galacturonic acid. Only statistically significant differences among genotypes indicated with stars and dots assessed for each individual sugar are shown above bars, as determined by ANOVA followed by Tukey's HSD test $(P<0.001)$. (F) Lignin deposition visualized by phloroglucinol stain (in fuchsia) in primary roots of indicated genotypes. (G to I) ESMD1p:NLS-3xVEN expression pattern in 5-do WT primary roots counterstained with propidium iodide. $(\mathrm{H})$ Orthogonal view from epidermis to vascular cylinder of a Z-stack section in the early differentiation zone through ( $G$, dotted line). (I) Increased magnification in the early differentiation zone from ( $G$, boxed). ep, epidermis; co, cortex; en, endodermis. Letters in ( $B, C, D)$ denote statistically significant differences among samples as determined by ANOVA followed by Tukey's HSD test $(P<0.05)$. Scale bars, $0.5 \mathrm{~mm}(\mathrm{~A}), 200 \mu \mathrm{m}(\mathrm{F}$ and $\mathrm{G}), 25 \mu \mathrm{m}(\mathrm{H})$, and $50 \mu \mathrm{m}(\mathrm{I})$. 
A

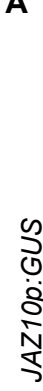

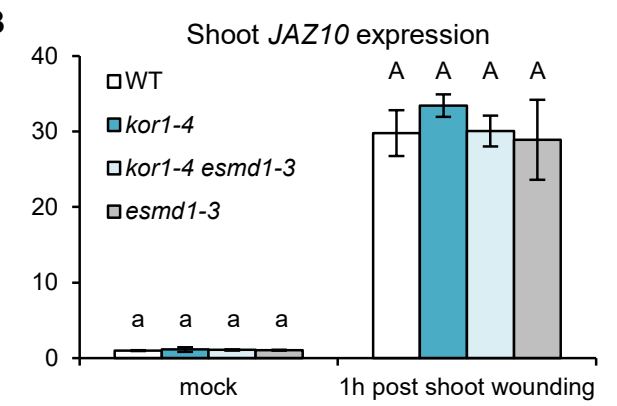

kor1-4 esmd1-3

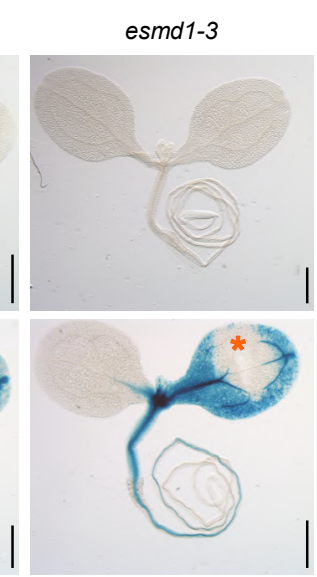

C

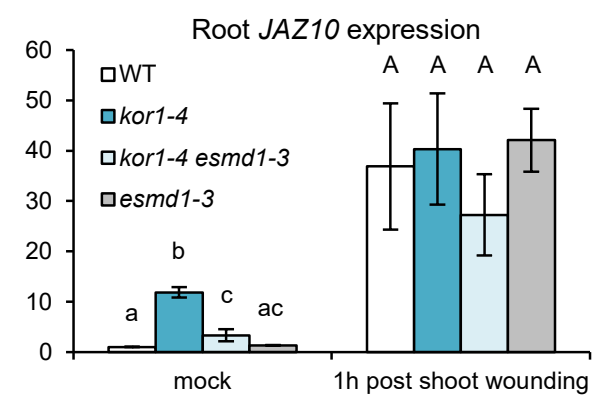

D
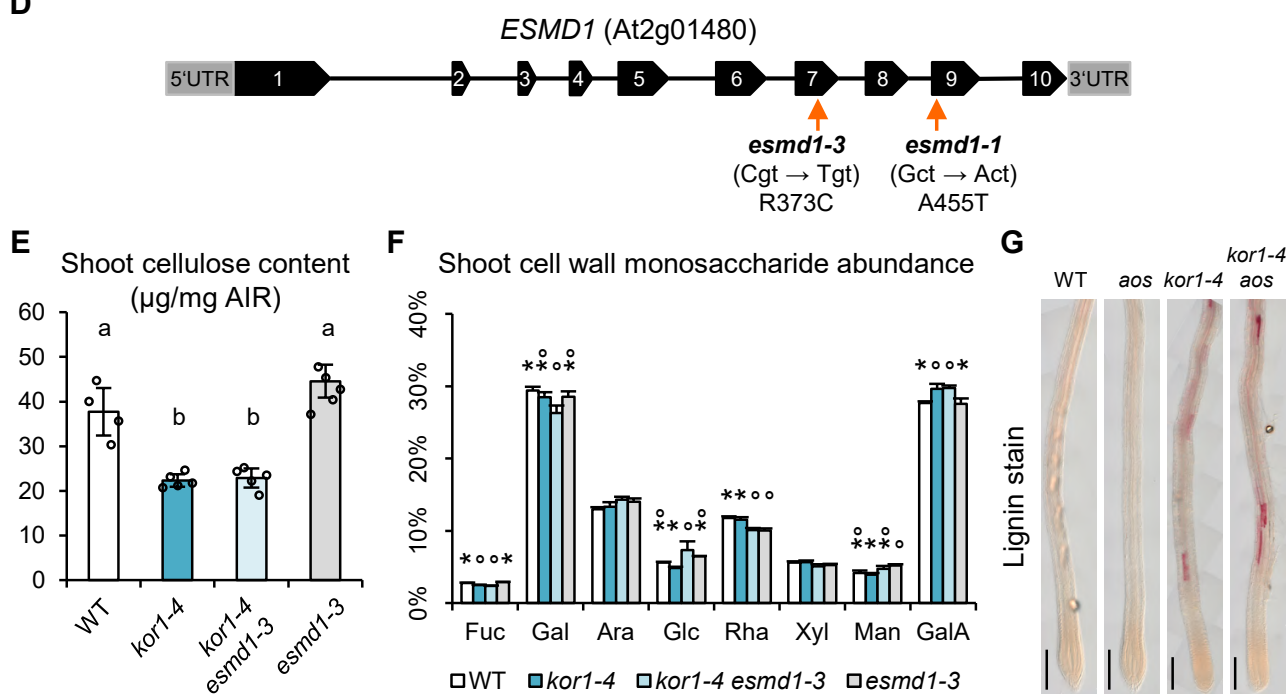

Fig. S4 ESMD1 regulates JA levels upon kor1-dependent cell wall changes in roots

(A) JAZ10p:GUS expression in kor1-4, kor1-4 esmd1-3, esmd1-3 and kor1-4 esmd1-3 complemented with ESMD1p:ESMD1- $m T$ at basal conditions and $2 \mathrm{~h}$ after cotyledon wounding (orange asterisks). (B and C) qRT-PCR of JAZ10 expression in (B) shoots and (C) roots of indicated genotypes basally and 1 $\mathrm{h}$ post cotyledon wounding. JAZ10 transcript levels were normalized to those of UBC21. Bars represent the means of 3 biological replicates $( \pm S D)$, each containing a pool of $\sim 60$ organs from 5 -do seedlings. (D) Schematic representation of ESMD1 gene structure describing the two esmd1 alleles used in this study. Black boxes depict exons and lines introns. (E) Crystalline cellulose content from alcohol insoluble residue (AIR) extracted from shoots of indicated genotypes. Bars represent means of five biological replicates depicted as circles $( \pm S D)$, each consisting of pools from 100 shoots from 12-do seedlings. (F) Cell wall monosaccharide composition analysis from AIR extracted from shoots of indicated genotypes. Bars represent the means of four biological replicates $( \pm S D)$, each containing a pool of 100 shoots from 12-do seedlings. Fuc, fucose; Gal, galactose; Ara, arabinose; Glc, glucose; Rha, rhamnose; Xyl, xylose; Man, mannose; GalA, Galacturonic acid. Only statistically significant 
differences among genotypes indicated with stars and dots assessed for each individual sugar are shown above bars, as determined by ANOVA followed by Tukey's HSD test $(P<0.001)$. (G) Phloroglucinol stain showing lignin deposition (in fuchsia) in primary roots of indicated genotypes. Letters in $(B, C$, and $E)$ denote statistically significant differences among samples as determined by ANOVA followed by Tukey's HSD test $(P<0.05)$. Scale bars, $0.5 \mathrm{~mm}(A)$, and $200 \mu \mathrm{m}(G)$.

\section{Enlarged kor1 cortex cells impact JA production in inner tissues}

Having found that ESMD1 expression includes our zone of interest, we further noticed that kor1-4 esmd1-3 roots are considerably thinner than kor1-4 (Fig. 4A and B). Transversal sections across the root early differentiation zone coinciding with the sites of JA production in kor1-4, revealed that root thickness in the mutant was twice that of the WT and resulted from enlarged areas of all cell-types examined (Fig. 4C and D, Fig. S5A to F). The relatively small expansion of kor1-4 epidermal cell area (1.1-fold) was counterbalanced by a significant increase in their cell number (Fig. S5A and C). Although aberrant cell divisions were also occasionally observed in kor1-4 cortex and endodermal cells, the overall cell number in these cell types did not differ significantly from the WT (Fig. S5A). The largest augmentation in kor1-4 cell area was observed in cortex cells, being 2.6-fold larger than WT, while endodermal and pericycle cells displayed an expansion of 2- and 2.4-fold, respectively (Fig. 4C and D, Fig. S5C to F). These kor1-4 phenotypes were almost completely restored to WT levels in the kor1-4 esmd1-3 suppressor, which showed a diminished root thickness, a full complementation of epidermal cell numbers, and reduced cell areas of cortex, endodermis and pericycle cells (Fig. 4A to D, Fig. S5A to F). Notably, the large expansion of kor1-4 cortex cells was drastically reduced in kor1-4 esmd1-3 (Fig. 4C and D), leading us to hypothesize that enlarged cortex cells may impinge upon inner tissues.

To verify if kor1-4 enlarged cortex cells indeed impacted JA-lle production in inner epidermal and pericycle, we analysed our transgenic lines expressing CIT-KOR1 under cell-type-specific promoters in transversal root sections coinciding with ectopic JA production (Fig. 2). As expected, expressing CIT-KOR1 under its native promoter fully restored kor1-4 root thickness to WT dimensions, while epidermis-, cortex- and endodermis-specific CIT-KOR1 expression reduced kor1-4 root thickness to various degrees. Stele-specific CIT-KOR1 expression (st-WOLp) did not have any significant effect and was thus excluded from further analysis (Fig. $4 \mathrm{~A}$ and B). Compared to the KOR1p:CIT-KOR1 complemented kor1-4 line, epidermal CIT-KOR1 expression (ep-IRT1p) still exhibited typical kor1-4 features of 2-fold enlarged root area, increased epidermal cell number, and enlarged areas of cortex, endodermal and pericycle cells (Fig. 4E and F, Fig. S5G to L). Conversely, expressing CIT-KOR1 in either cortex (co-PEPp) or endodermal (en-SCRp) cell layers, rendered kor1-4 phenotypes more similar to the KOR1p:CIT-KOR1 complemented kor1-4 line by showing a 1.4-fold increase in total root 
area, and only a milder enlargement of cortex, endodermis, and pericycle cells (Fig. 4E and F, Fig. S5G to L). While the most prominent effect of expressing CIT-KOR1 in cortex or endodermis was to reduce the area of cells in which the fusion protein was localized, the strongest correlation between JA signalling and cell-type area was again found for the cortex cell layer. In fact, expressing CIT-KOR1 in the cortex (co-PEPP) abolished kor1-4 JGP expression, restored cortex cell area to almost WT levels (1.2-fold) without fully recovering cellular enlargement of endodermal and pericycle cells which persisted being 1.6- and 1.7-fold larger than the KOR1p:CIT-KOR1 complemented line (Fig. 2E, 4E and F). In turn, endodermal CIT-KOR1 expression (en-SCRp) did not abolish elevated JA levels in kor1-4 nor led to a drastic reduction in cortex expansion which remained 1.7-fold larger, albeit almost completely restoring endodermal and pericycle cell areas (Fig. 2E, 4E and F). As KOR1 activity in cortex cells is important to regulate their size and JGP expression in adjacent inner tissues, and ESMD1 is expressed in both cortex and endodermal cells, it is conceivable that the cortexendodermis interface is critical for governing JA-lle production in kor1.

A

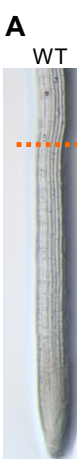

C WT

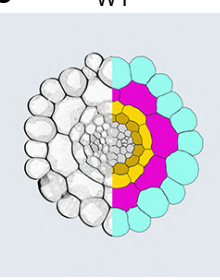

E

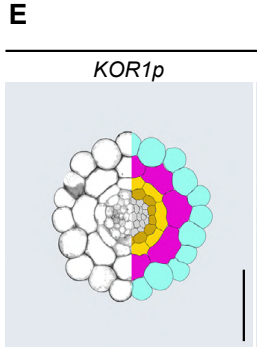

kor1-4

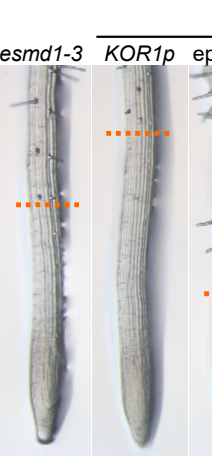

kor1-4

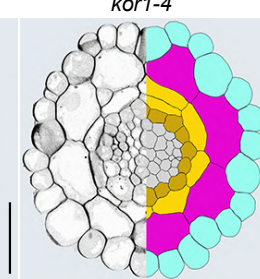

CIT-KOR1 in kor1-4

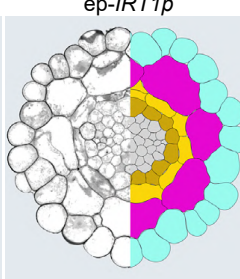

kor1-4 esmd1-3

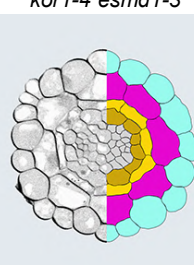

co-PEPp

CIT-KOR1 in kor1-4

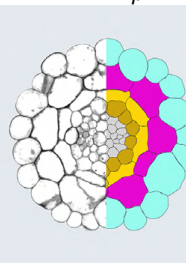

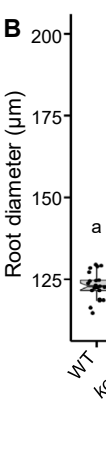

esmd1-3
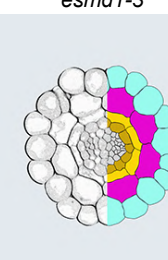

en-SCRp

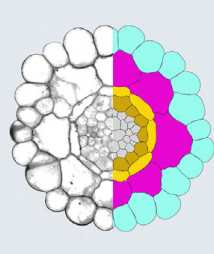

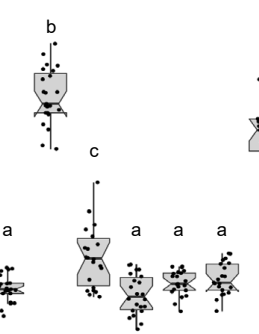

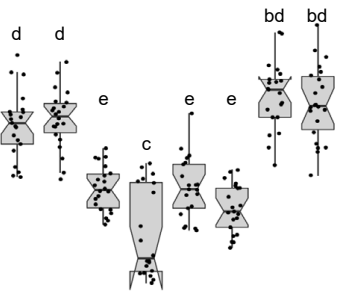

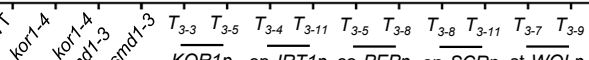

की

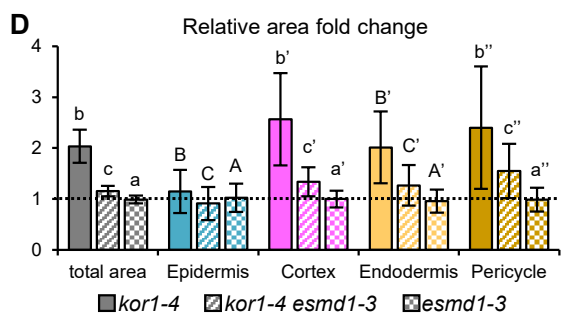

$\mathbf{F}$

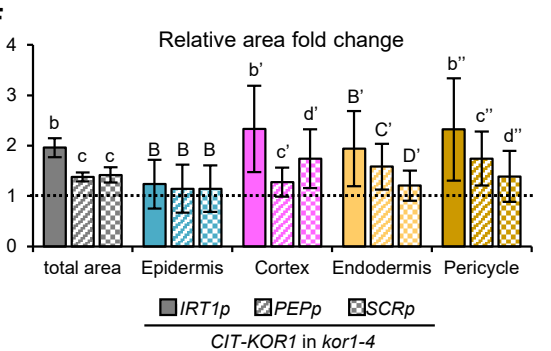

Fig. 4 Cortex cell enlargement in kor1 correlates with constitutive JA production.

(A) Representative primary root images of WT, kor1-4, kor1-4 esmd1-3, esmd1-3 and kor1-4 complemented with CIT-KOR1 under the control of its native KOR1p promoter, or epidermis- (epIRT1p), cortex- (co-PEPp), endodermis- (en-SCRp) or stele-specific (st-WOL1p) promoters. Orange dashed lines define the initiation of the differentiation zone, as indicated by the appearance of root hairs in 7-do seedlings. (B) Box plot summary of primary root diameter at the onset of differentiation 
in 7-do seedlings of indicated genotypes. Medians are represented inside the boxes by solid lines, circles depict individual measurements $(n=22-23)$. ( $C$ to $\mathbf{F}$ ) Anatomy and cell size comparisons from transverse sections across the early differentiation zone of the primary root in (C and D) WT, kor1-4, kor1-4 esmd1-3 and esmd1-3, and in (E and F) kor1-4 complemented with cell-type-specific promoters. ( $C$ and $E$ ) Representative split images from cross sections (left panels) and respective cell segmentations (right panels). Segmented cell-types are color-coded as: epidermis, turquoise; cortex, magenta; endodermis, yellow; pericycle, mustard; stele, grey. ( $D$ and F) Fold change in total and cellspecific areas from segmented transversal root sections in indicated genotypes. Measurements were normalized to those of the (D) WT or (F) KOR1p:CIT-KOR1, indicated by dashed lines (individual measurements are available in Fig. S5). Letters in (B, D, F) denote statistically significant differences among samples as determined by ANOVA followed by Tukey's HSD test $(P<0.05)$. Scale bars, 200 $\mu \mathrm{m}(\mathrm{A}), 50 \mu \mathrm{m}$ ( $\mathrm{C}$ and $\mathrm{E})$.
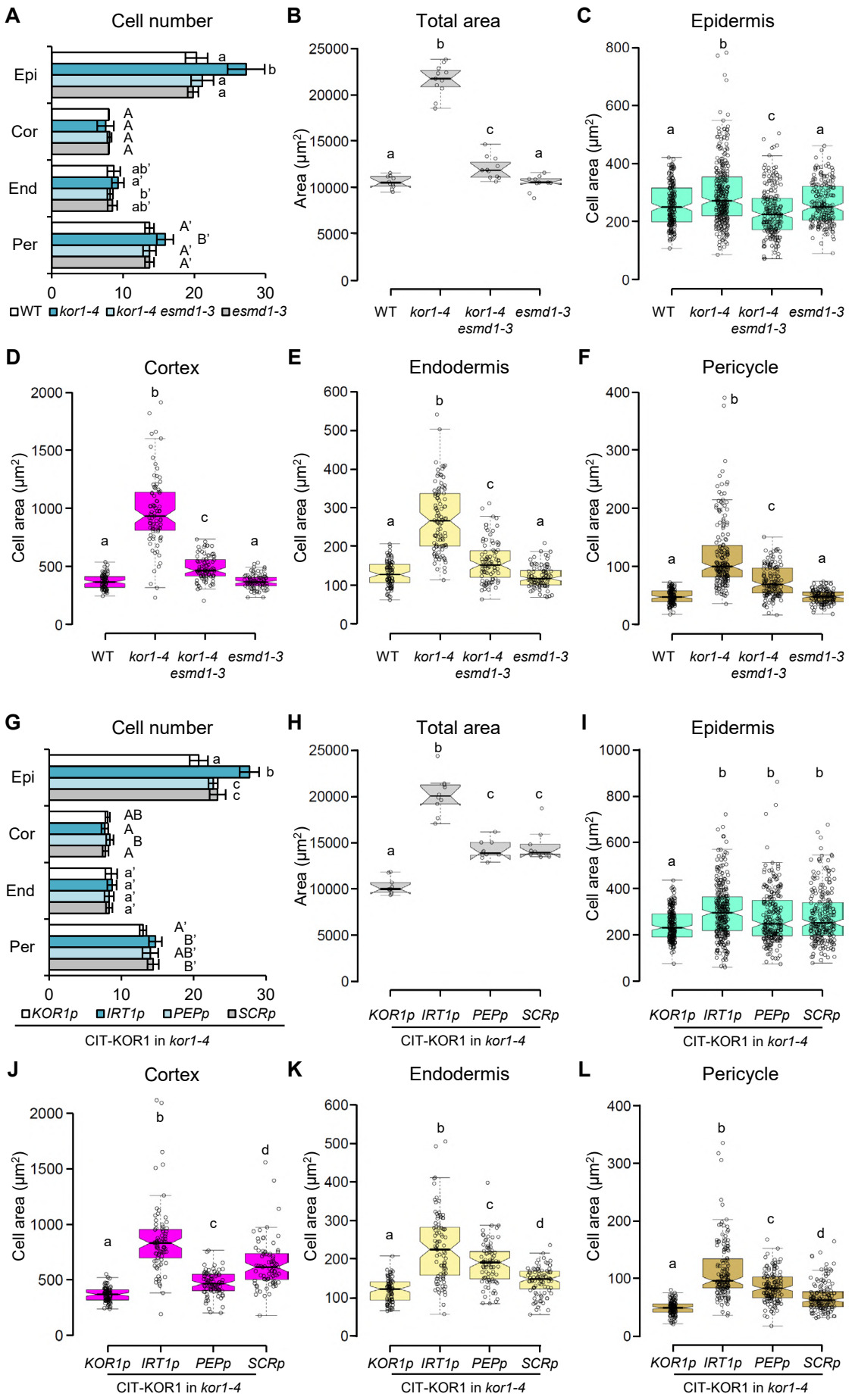
Fig. S5 Cellular measurements in transverse root sections of kor1, its suppressor kor1 esmd1, and its cell-type specific CIT-KOR1 complementation lines.

(A) Cell number in epidermis (Epi), cortex (Cor), endodermis (End) and pericycle (Per) of the early differentiation zone of primary roots in WT, kor1-4, kor1-4 esmd1-3 and esmd1-3 5-do seedlings. (B to F) Box plot summary of (B) primary root total area, and cell-type specific areas in (C) epidermis, (D) cortex, (E) endodermis, and (F) pericycle cells from transverse sections of the early differentiation zone in WT, kor1-4, kor1-4 esmd1-3and esmd1-3 5-do seedlings. (G) Cell number in individual tissue layers of the early differentiation zone of primary roots in 5-do kor1-4 seedlings complemented with KOR1p:CIT-KOR1 or CIT-KOR1 expressed in epidermis (IRT1p), cortex (PEPp) or endodermis (SCRp) cells. ( $\mathbf{H}$ to $\mathbf{N}$ ) Box plot summary of $(\mathrm{H})$ primary root total area, and cell-type specific areas in (I) epidermis, $(\mathrm{J})$ cortex, $(\mathrm{K})$ endodermis, and $(\mathrm{L})$ pericycle cells from transverse sections of the early differentiation zone in 5-do kor1-4 seedlings complemented with cell-type specific promoters. Medians are represented inside the boxes by solid lines, circles depict individual cell measurements from 10-11 roots. Letters denote statistically significant differences among samples as determined by ANOVA followed by Tukey's HSD test $(P<0.05)$.

\section{Osmotic support abolishes constitutive JA production in kor1}

JA responses can be triggered by exogenous treatment with specific pectin- and cellulose-derived fragments serving as ligands binding putative plasma membrane receptors and activating intracellular signalling, reviewed in (11). More notoriously, JA-lle biosynthesis is swiftly activated by mechanical stress (24). The lack of a clear association between cell wall composition and JA production in kor1 (Fig. 3, Fig. S4) led us to hypothesize that increased JA-lle levels in kor1 may result from enlarged cortex cells 'squeezing' spatially constrained inner tissues, rather than to cell wallderived elicitors. To test this hypothesis, we decreased intracellular turgor pressure by growing kor14 seedlings under hyperosmotic conditions to withdraw water from their cells. All tested substances acting as osmotica (mannitol, sorbitol, PEG, hard agar) effectively abolished JA signalling in kor1-4 roots (Fig. 5A and B, Fig. S6A). Mannitol-grown seedlings still responded to wounding, reinforcing the tissue-specificity of the JGP reporter (Fig. S6B). Furthermore, while WT root length decreased under hyperosmotic conditions, kor1-4 root elongation exhibited a tangible amelioration (Fig. 5C). This was also reflected in the reduced area of kor1-4 transversal root sections grown on mannitol reverting to WT size (Fig. 5D and E). Consistently, mannitol treatment restored kor1-4 epidermal cell number as well as enlarged epidermal, cortex, endodermis and pericycle cell size (Fig. 5D and E, Fig. $\mathrm{S} 6 \mathrm{C}$ to $\mathrm{H})$. Notably, the hyperosmotic treatment fully recovered the enlarged kor1-4 cortex cell area to WT levels, while endodermal and pericycle cell were still 20\% larger with respect to the WT (Fig. $5 \mathrm{D}$ and $\mathrm{E})$. Thus, the activation of JA production in kor1 is consistent with inner tissues being mechanically stressed by enlarged cortex cells. 

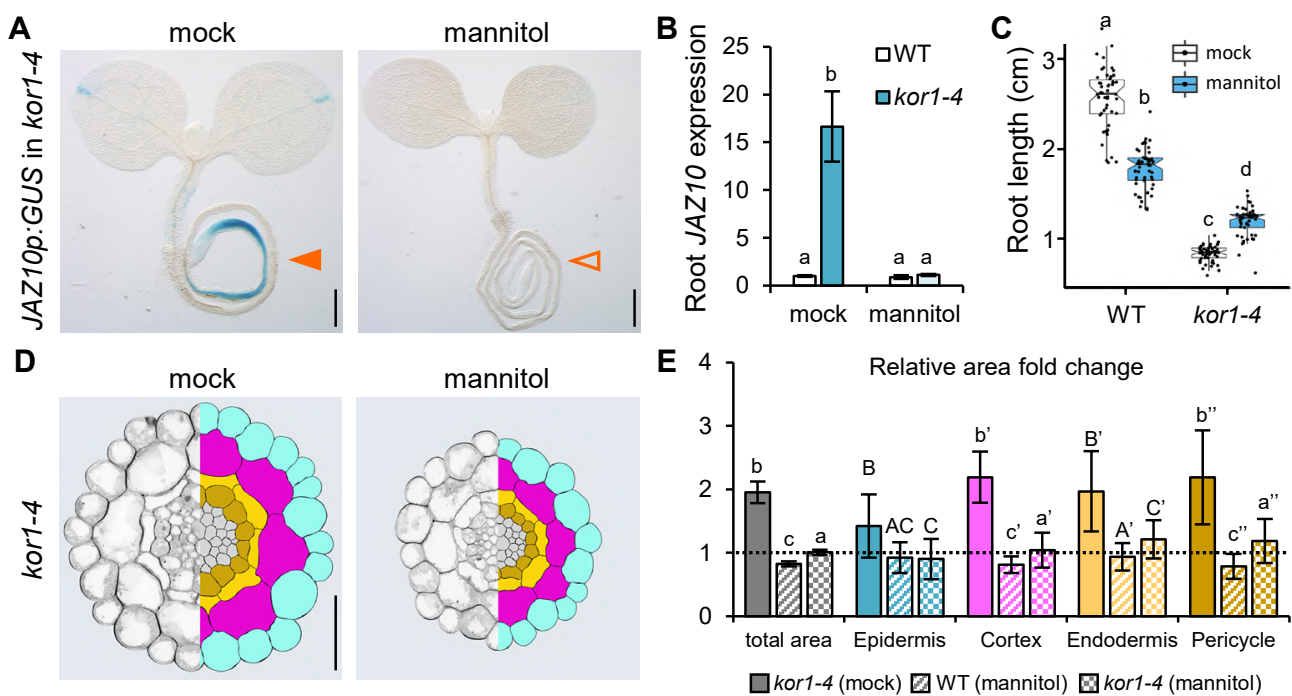

Fig. 5 Hyperosmotic mannitol treatment relieves JA signalling in kor1 to WT levels.

(A) JAZ1Op:GUS reporter activity in 5-do kor1-4 seedlings grown in mock or 3\% mannitolsupplemented media. Note the presence of ectopic JA signalling in mock conditions (orange arrowhead) and its abolishment in mannitol (empty arrowhead). (B) qRT-PCR of basal JAZ10 root expression of WT and kor1-4 grown in the presence or absence of 3\% mannitol. JAZ10 transcript levels were normalized to those of UBC21. Bars represent the means of 3 biological replicates ( $\pm S D)$, each containing a pool of $\sim 60$ organs from 5-do seedlings. (C) Primary root length box plot summary of 7-do WT and kor1-4 seedlings grown in the presence or absence of $3 \%$ mannitol. Medians are represented inside the boxes by solid lines, circles depict individual measurements ( $n=59-61$ ). A linear model was used to assess differences in responsiveness between the two genotypes: WT plants responded with a decrease in root length $(-0.78 \mathrm{~cm}$; $p$-value: $2 \mathrm{e}-16)$, whereas kor1-4 with an increased $(+0.35 \mathrm{~cm}$; p-value: $2 \mathrm{e}-16)$. (D) Representative split images from transverse sections (left panels) and respective cell segmentations (right panels) across the early differentiation zone of primary WT and kor1-4 roots grown in the absence (mock) or presence of $3 \%$ mannitol. Segmented cell-types are color-coded as: epidermis, turquoise; cortex, magenta; endodermis, yellow; pericycle, mustard; stele, grey. (E) Fold change in total and cell-type specific areas from segmented transverse sections of WT and kor1-4 grown with and without 3\% mannitol. Measurements were normalized to those of the mock-treated WT indicated by a dashed line (individual measurements are available in Fig. S6). Letters in ( $B, C$, and $E$ ) denote statistically significant differences among samples as determined by ANOVA followed by Tukey's HSD test $(P<0.05)$. Scale bars, $0.5 \mathrm{~mm}(\mathrm{~A})$, and $50 \mu \mathrm{m}$ (D). 
A
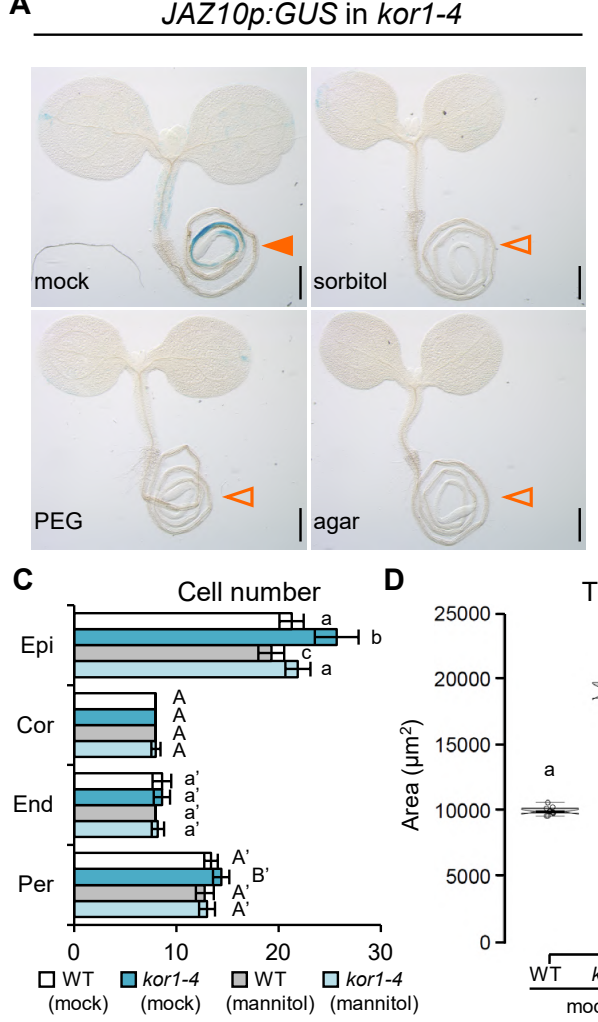

$\mathbf{F}$

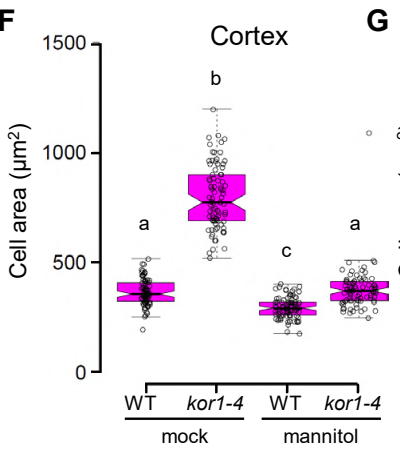

B

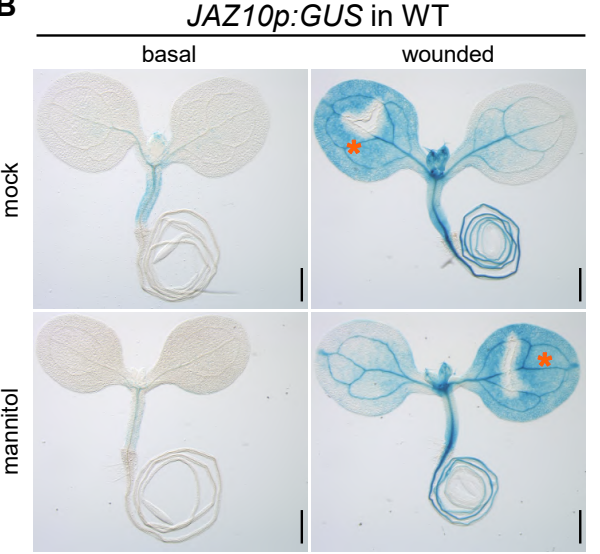

E
Epidermis

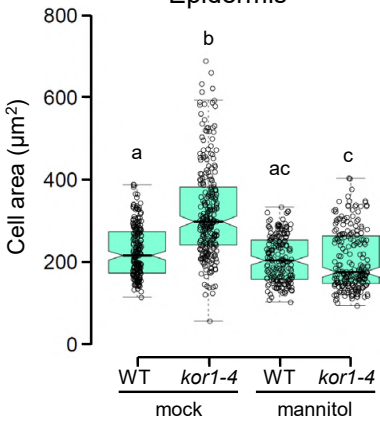

H

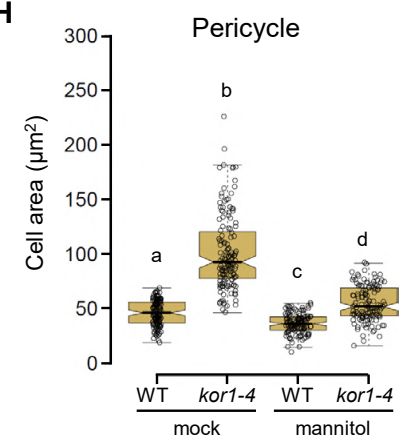

Fig. S6 Hyperosmotic treatments and cellular measurements.

(A) Basal JAZ10p:GUS reporter activity in 5-do kor1-4 seedlings grown in the absence (mock) or presence of $3 \%$ sorbitol, $3 \%$ PEG or in $3 \%$ agar. Note the presence of ectopic JA signalling in mock conditions (orange arrowhead) and its abolishment in hyperosmotic media (empty arrowhead). (B) JAZ10p:GUS reporter activity in 5-do WT seedlings grown in the absence (mock) or presence of $3 \%$ mannitol under basal conditions and $2 \mathrm{~h}$ after cotyledon wounding (orange asterisks). Scale bars, 0.5 $\mathrm{mm}$ (A and B). (C) Cell number in individual tissue layers of the early differentiation zone of 5-do primary roots of WT and kor1-4 seedlings, grown in the absence or presence of $3 \%$ mannitol. (D to H) Box plot summary of (D) primary root total area, and cell-type specific areas of (E) epidermis, (F) cortex, $(\mathrm{G})$ endodermis, and $(\mathrm{H})$ pericycle cells from transversal cross sections of the early differentiation zone in WT and kor1-4 5-do seedlings, grown in the absence or presence of 3\% mannitol. Medians are represented inside the boxes by solid lines, circles depict individual cell measurements from 10 roots. Letters denote statistically significant differences among samples as determined by ANOVA followed by Tukey's HSD test $(P<0.05)$. 


\section{Heightened JA levels guide kor1 roots towards greater water availability}

The activation of JA signalling often leads to an induction in defense responses accompanied by a reduction in organ growth (37). It is thus possible that increased JA production in kor1 contributes to slow down the mutant's root growth rate to better adjust to cellulose deficiency, and concomitantly constitutively protect the mutant from potential threats found in the soil. However, blocking JA production in kor1-4 did not alter root elongation nor the expression of defense marker genes, as the mutant had the same root growth rate as its JA-deficient kor1-4 aos counterpart and similar low abundance of VSP2 and PDF1.2 transcripts (Fig. 6A, Fig. S7A and B). What could then be the consequence of increased JA signalling in kor1?

As the stunted kor1-4 root growth was alleviated by continuous growth in mannitol (Fig. 5C), we tested if the mutant might preferentially grow towards hyperosmotic conditions in a split-agar assay used for measuring root hydrotropic responses among genotypes elongating at similar growth rates (38). Following transfer to split-agar plates with mannitol harbouring asymmetric water availability, both WT and aos seedling roots readjusted their growth away from the hyperosmotic media in search for greater water availability (Fig. 6B, Fig. S7C and D). Contrary to our expectations, kor1-4 roots also bent away from mannitol with a positive hydrotropic root curvature despite their slower growth rate. To our surprize, the JA-deficient kor1-4 aos double mutant failed to effectively readjust its root growth direction towards a greater water availability and instead grew into the mannitol media (Fig. 6C, Fig. S7E). This effect was not a consequence of the double mutant's inability to undergo general root bending responses such as those triggered by root gravitropism, but rather to a specific insensitivity to root hydrotropism (Fig. S7F to H). Our data thus unveiled that the constitutive activation of JA-Ile production and signalling in kor1-4 serves to facilitate water foraging. 


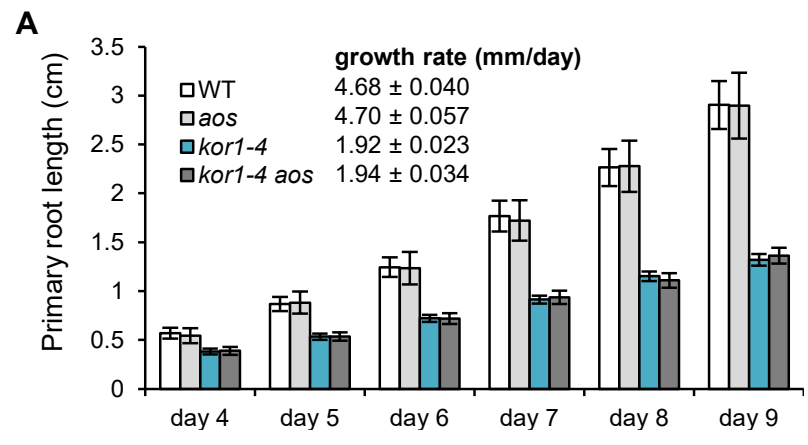

B
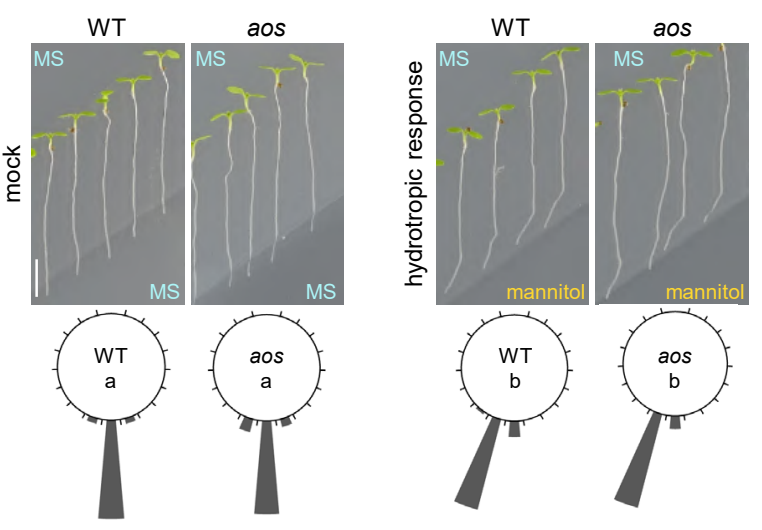

C
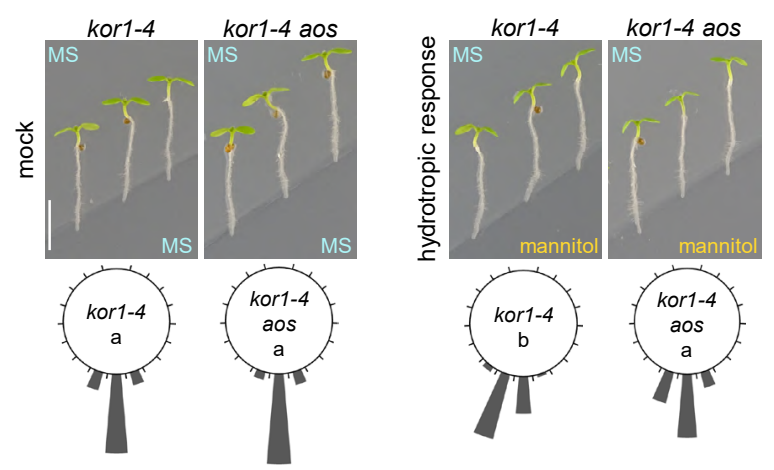

Fig. 6 Increased JA production facilitates kor1 response to root hydrotropism.

(A) Primary root elongation of indicated genotypes between 4- and 9-days post germination. Bars represent the means of 40-50 plants. Data were used to determine the root growth rate in $\mathrm{mm}$ per day by linear regression. (B and C) Root hydrotropic response of (B) WT and aos, and (C) kor1-4 and kor1-4 aos seedlings. Representative images and circular histograms summarizing root curvatures of indicated genotypes $24 \mathrm{~h}$ after transfer to split-agar Murashige and Skoog (MS) plates under mock (MS/MS) or hydrotropism-inducing (MS/400 mM mannitol) conditions. Bars indicate the percentage of seedlings exhibiting a root bending angle assigned to one of the $1820^{\circ}$ sectors on the circular diagram (individual measurements are available in Fig. S7D and $E$ ), $n=42$. Letters indicate statistically significant differences as determined by Two-Way-ANOVA followed by Tukey's HSD test $(P<0.05)$. Scale bar, $5 \mathrm{~mm}$. 
A

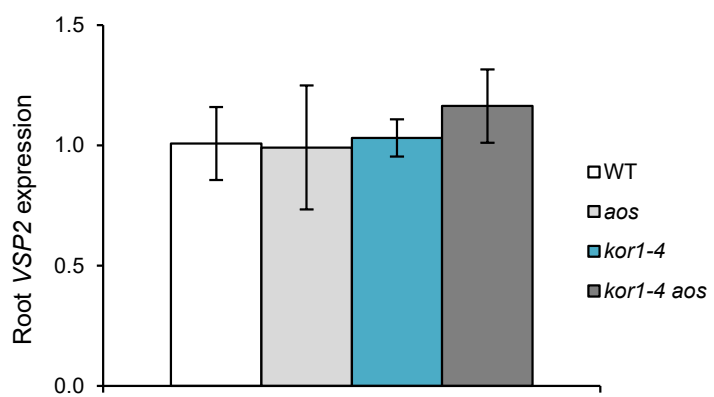

C

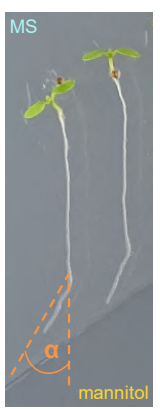

F

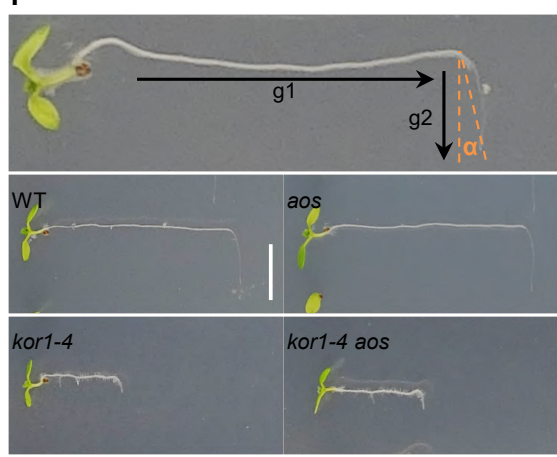

D

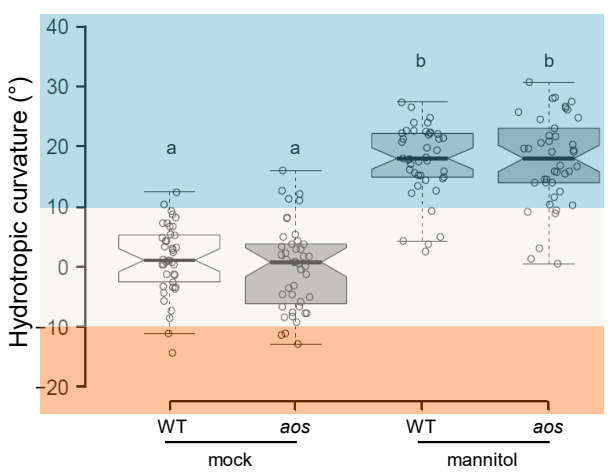

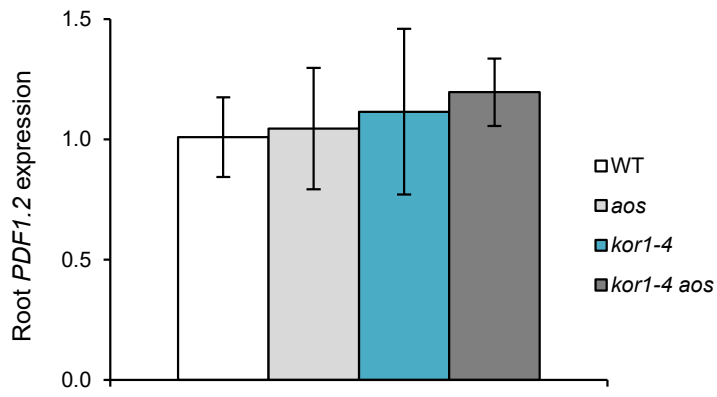

E
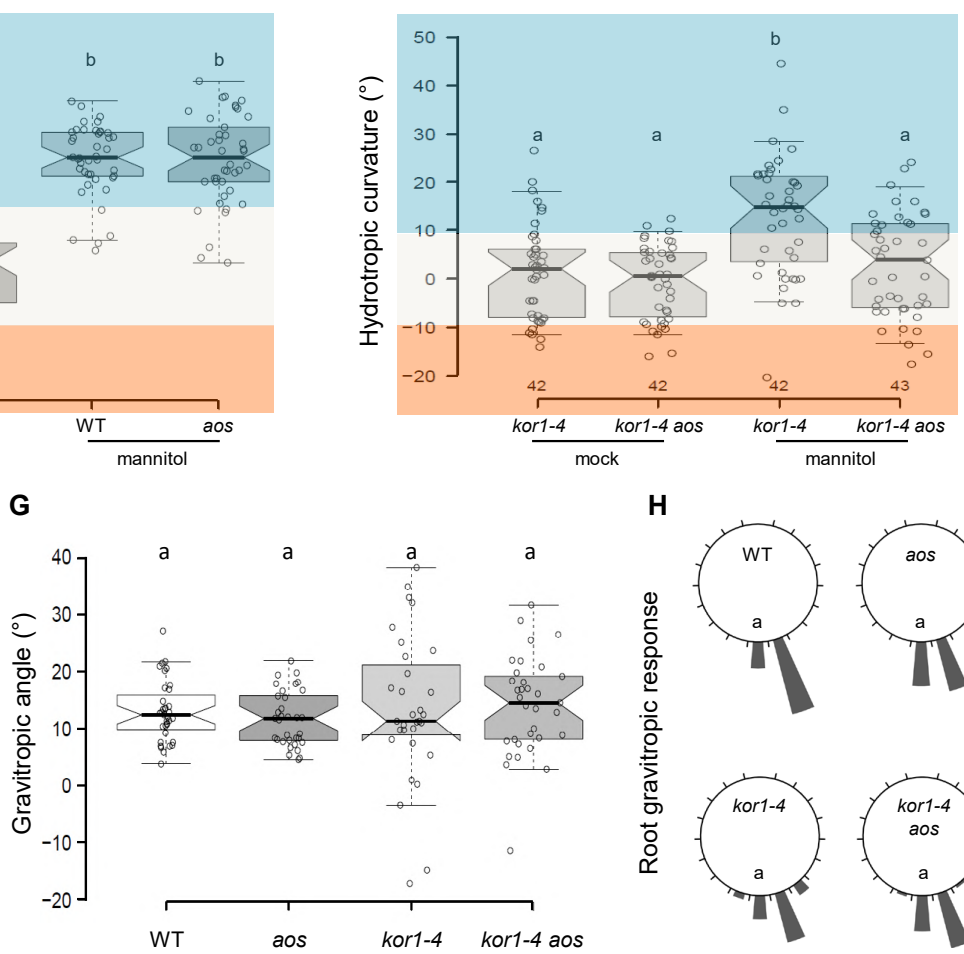

H

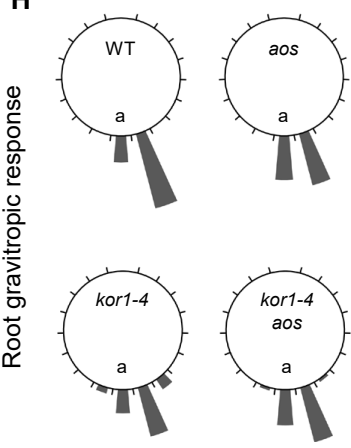

Fig. S7 Elevated JA levels in kor1 do not alter defense marker gene expression nor root gravitropism.

(A and B) qRT-PCR of (A) VSP2 and (B) PDF1.2 basal levels in roots of indicated genotypes. Transcript levels were normalized to those of $U B C 21$ and displayed relative to the WT controls. Bars represent the means of 3 biological replicates $( \pm S D)$, each containing a pool of $\sim 60$ organs from 5 -do seedlings. ( $C$ to E) Hydrotropic root curvature in seedlings grown with similar growth rates: WT and aos; and kor1-4 and kor1-4 aos. (C) Representative image of a WT seedling $24 \mathrm{~h}$ after transfer to a split-agar plates under hydrotropism-inducing (MS/400 mM mannitol) conditions depicting the measurement of its hydrotropic root curvature angle $(\alpha)$. Box plot summary of root hydrotropic curvatures in (D) WT and aos, and (E) kor1-4 and kor1-4 aos seedlings $24 \mathrm{~h}$ after transfer to split-agar (MS/MS, mock) or hydrotropism-inducing (MS/400 mM mannitol) plates. Medians are represented inside the boxes by solid lines, circles depict individual measurements $(n=42)$. Hydrotropic responses were highlighted as positive (blue, towards greater water availability), neutral (light grey, straight growth) and negative (orange, towards lower water availability) along the y-axis. (F to $\mathbf{H}$ ) Root gravitropic response of indicated genotypes. (F) Representative images depicting seedlings grown vertically for 5 -d in the $1^{\text {st }}$ gravity direction (g1), turned by $90^{\circ}$ and grown for additional $24 \mathrm{~h}$ in the $2^{\text {nd }}$ gravity vector (g2) before measuring the gravitropic root curvature angle $(\alpha)$. (G) Box plot summary of the root gravitropic angle of WT, aos, kor1-4 and kor1-4 aos seedlings. Medians are represented inside the boxes by solid lines, circles depict individual measurements $(n=31-35)$. $(H)$ Circular histograms summarizing root growth curvatures of indicated genotypes from (G). Bars indicate the percentage 
of seedlings exhibiting a root bending angle assigned to one of the $1820^{\circ}$ sectors on the circular diagram. Letters in $(D, E, G$, and $H)$ indicate statistically significant differences as determined by TwoWay-ANOVA followed by Tukey's HSD test $(P<0.05)$.

\section{DISCUSSION}

Plant cell walls are defining plant structures which serve a wide range of biological functions including shaping cell morphology, providing mechanical support for growth, and connecting cells within tissues. Changes in their structure and assembly may have pronounced repercussions on intracellular as well as whole-plant responses (21). Here we investigated how cues derived from perturbed cell walls integrate with the production of the stress hormone JA-lle. The identification of cellulose-deficient kor1 alleles displaying elevated JA-lle levels specifically in seedling roots prompted us to examine cell wall-triggered induction of hormone biosynthesis in cell-type-specific contexts. By restoring KOR1 function in specific layers of the primary root, we showed that ectopic JA-lle signalling in endodermal and pericycle cells was caused by cell non-autonomous signals deriving from adjacent and markedly enlarged cortex cells. Swollen kor1 cortex cells could exert an increased mechanical pressure upon both external epidermal cells as well as inner tissues. As JA-Ile production is readily triggered by mechanical stress, why was the hormone increase observed only in inner tissues? A possibility is that epidermal cells could dissipate the cortex-exerted mechanical pressure by expanding towards the available outer space and by stimulating cell division. Mechanical cues are known to influence cell cycle progression in animals, and although this has not been demonstrated in plants yet, they guide the orientation of plant cell division planes $(20,39)$. Contrary to outer tissues, endodermal and pericycle cells are physically constrained and may become mechanically compressed by enlarged cortex cells. Indeed, reducing the swollen kor1 cortex cell size in three independent manners fully abolished increased JA-lle signalling in inner tissues. First, restoring KOR1 activity in the cortex complemented both cortex cell size and JA-lle marker gene expression. Second, we identified a genetic suppressor of kor1 in which cortex cell size and JA levels were also recovered. Third, by reducing kor1 turgor pressure with hyperosmotic treatments, enlarged cortex cells and JA signalling were fully recovered. Collectively, the data demonstrate that the JA pathway is triggered by turgor pressure-driven mechanical changes in response to cellulosedeficiency. Our findings might explain why wounding single-cells by laser ablation in living roots does not induce JA-lle signalling (27). The disintegration of a target cell provokes adjacent cells to bulge towards the vacuity left by the ablated cell, in a process compatible to turgor pressure changes in adjacent cells due to the vanished support from the damaged cell $(40,41)$. However, similarly to epidermal cells in kor1, wound-adjacent cells are not subjected to compression and might be able to compensate the increased mechanical stress by expanding towards the void space without 
activating JA production. On the other hand, mechanical wounding and chewing insect herbivores squash tissues and possibly compress spatially constrained cells adjacent to wounds. Because cells within tissues are connected through their cell walls, mechanical stress signals arising from turgor pressure changes may propagate over distances depending on the extent of damage (42). Consistently, it has been proposed that mechanical tissue damage causes sudden pressure changes in vascular xylem vessels, compressing cells adjacent to vessels where JA biosynthesis is then initiated $(24,43)$. Hence, mechanical compression generating turgor pressure changes may be a crucial elicitor of JA-lle biosynthesis in circumstances extending beyond cell wall perturbations, with putative osmo- and mechano-sensors located across different subcellular compartments (44). However, measuring mechanical stress patterns in vivo, particularly from cells embedded in inner tissues which are not physically accessible, remains challenging (45). Emerging tools are offering encouraging prospects to assess mechanobiological processes in vivo in future studies (46).

In our search to identify genetic regulators of ectopic JA-lle production in kor1 with potential roles in stress signals arising from turgor pressure changes, we did not retrieve known components involved in osmoregulation such as mechanically activated ion channels OSCA1 or MSL10, nor cell wall integrity sensors such as THESEUS1 whose loss of function was insensitive to isoxaben-induced JA production $(25,47-49)$. This might be due to the screen not being saturated or to genetic redundancy. Instead, the kor1 suppressor screen recovered an allele in ESMD1, a putative $O$-fucosyltransferase involved in pectin homeostasis (31). Although ESMD1's substrate and biochemical function are unknown, it was proposed that it may $O$-fucosylate cell wall integrity sensor proteins and thereby contribute to cell signalling (31). Recently, two enzymes belonging to the same GT106 family were shown to be rhamnosyltransferases involved in the biosynthesis of the pectin component rhamnogalacturonan I (RG-I) $(32,50)$. Given that our monosaccharide composition analysis in genotypes with esmd1-3 showed a substantial rhamnose reduction which in Arabidopsis derives mostly from RG-I (51), and in agreement with other GT106 family members, it is possible that ESMD1 is directly involved in pectin biosynthesis. Although it remains unclear how esmd1 restores cell shape and JA-Ile levels in kor1, RG-I can have significant and specific contacts with cellulose microfibrils (52). As turgor pressure is counterbalanced by cell walls being under tension (53), it is possible that the diminished cell wall tensile strength in kor1 is compensated by lack of ESMD1. The identification of ESMD1 also indicates that cell size and mechanical compression on inner tissues can be alleviated by remodelling cell wall properties and thus re-equilibrate turgor pressure. 
A remarkable observation was that constitutive JA-lle signalling enabled kor1 roots to modify their growth towards greater water availability. This finding highlights the importance of characterizing cell wall mutants as they may provide opportunities to uncover more subtle functions of the JA pathway. While the root hydrotropic response of the JA-deficient aos mutant did not differ from the WT, JA-deficiency in kor1 prevented roots bending away from the media with reduced water availability. These findings imply that while JA-deficiency per se does not impact root hydrotropism, the constitutive activation of JA-lle signalling in precise tissues and cell types is beneficial for root water foraging. Importantly, the constitutive activation of JA signalling in kor1 did not result in typical growth-inhibition responses $(54,55)$, suggesting that manipulating JA biosynthesis in specific cell-types might confer acclimation advantages without hampering overall growth. Interestingly, hyperosmotic mannitol treatments are also used to study plant responses to water deficiency, and applying exogenous JA to various plants renders them more tolerant to drought, reviewed in (56). Accordingly, Arabidopsis mutants impaired in JA production or signalling, are more droughtsusceptible (57). Although it is unknown how constitutive JA signalling guides kor1 roots away from mannitol, future studies deciphering the molecular mechanisms governing this effect could have beneficial impacts on breeding programs aimed at increasing plant drought tolerance.

\section{MATERIALS AND METHODS}

\section{Plant material and growth conditions}

Arabidopsis thaliana ecotype Columbia (Col) was used for all experiments. JAZ10p:GUS (JGP) transgenic lines in Col (WT) and in the JA-deficient aos mutant background were described previously (6). JAZ10p:NLS3XVEN was as in (27) but cloned and crossed into aos de novo. kor1-6 (SALK_075812) was obtained from NASC and crossed into JGP and aos JGP. esmd1-1 was described in (31). For in vitro assays, seeds were sterilized and stratified 2 days at $4{ }^{\circ} \mathrm{C}$ in the dark. Seedlings were grown on $0.5 x$ solid Murashige and Skoog (MS, Duchefa) medium supplemented with $0.5 \mathrm{~g} / \mathrm{L}$ MES hydrate (Sigma) and $0.7 \%$ or $0.85 \%$ plant agar (AppliChem) for horizontal or vertical growth, respectively. Horizontally grown seedlings were germinated on a nylon mesh placed on top of the MS media as described (6). Controlled growth conditions were set at $21^{\circ} \mathrm{C}$ under $100 \mu \mathrm{E} \mathrm{m} \mathrm{s}^{-1}$ light, with a $14 \mathrm{~h}$ light/10 $\mathrm{h}$ dark photoperiod. For soil propagation, transformation and crossing, plants were grown with the same temperature $(T)$ and light intensity, but under continuous light.

\section{Histochemical detection of GUS activity and lignin deposition}

GUS stainings were performed as described (58) and seedlings were photographed with a Leica M165 FC stereomicroscope fitted with a Leica MC170 HD camera. Lignin deposition was visualized 
by submerging seedlings in acidified phloroglucinol solution (1\% phloroglucinol in $18 \% \mathrm{HCl}$ ) for 5 min, washing in $1 \times$ PBS buffer, mounting in 10\% glycerol, and imaging using DIC optics on a Leica DM6B microscope fitted with a Leica DMC6200 camera.

\section{Plant treatments}

Single cotyledon wounding of seedlings and MeJA (Sigma) treatments to assess JGP reporter activity were performed as described (6). To evaluate the JA response of the JAZ1Op:NLS-VEN reporter in individual seedlings grown vertically, primary roots were mounted in mock or $10 \mu \mathrm{M}$ MeJA $0.5 \mathrm{x} \mathrm{MS}$ with $30 \mu \mathrm{g} / \mathrm{ml}$ propidium iodide ( $\mathrm{PI}$, Sigma) solution, imaged immediately $(\mathrm{t}=0)$ and after $2 \mathrm{~h}(\mathrm{n}=10)$. For osmotic support experiments, plant growth media was supplemented with either $3 \%$ mannitol (165mM, J\&K Scientific), 3\% sorbitol (165mM, Carl Roth), 3\% PEG6000 (Serva) or 3\% plant agar (AppliChem).

\section{Gene expression analyses}

RNA extraction and qRT-PCR of JAZ10 (At5g13220), JOX3 (At3g55970), VSP2 (At5g24770) and UBC21 (At5g25760) was performed as described $(29,58)$. Similarly, the expression of JAZ3 (At3g17860) was assayed with GACTCGGAGCCACAAAAGC and TACGCTCGTGACCCTTTCTTTG, and of PDF1.2 (At5g44420) with TTTGCTGCTTTCGACGCAC and GCATGATCCATGTTTGGCTCC primers. RT-PCR of KOR1 was performed with the primer pair AGATGCTGAAGCCAGAGCAG and TGTCATGGAGAGGTAATTCTGG.

\section{JA-lle quantification}

5-do roots were excised beneath the collet region and flash frozen to yield approximately $50 \mathrm{mg}$ FW for each biological replicate. Extraction and quantitative measurements were performed as described (29). The limit of JA-lle quantification ( $L O Q=3 x$ limit of detection) was determined from an Arabidopsis matrix as $0.49 \mathrm{pmol} / \mathrm{g} \mathrm{FW}$.

\section{Cloning and generation of transgenic lines}

All transcriptional and translational reporter constructs were generated by double or triple Multisite Gateway Technology (Thermo Fisher). ENTRY plasmids containing cell-type specific promoters ( $p E N-$ L4-IRT1p-L3, pEN-L4-PEPp-L3, pEN-L4-SCRp-L3, and pEN-L4-WOLp-L3) were described in (30) and obtained from NASC. pEN-L4-JAZ10p-R1, pEN-L1-NLS-3xVEN-L2 and pEN-R2-CIT-L3 were as in $(6,58)$. $\mathrm{CIT}$ and mTurquoise (mT) fluorophores were subcloned into pDONR221 or pDONR-P2R-P3 to obtain pEN-L1-CIT-L2 and pEN-R2-mT-L3. Promoters were amplified from WT genomic DNA with 
oligonucleotides containing adequate restriction sites for KOR1p (GATGATGCTCTCTGATAAAGC and AAGTCTTTTGGGAGCTGCAA, $2.132 \mathrm{~kb}$ ) and ESMD1p (ATCGACAGATCTCAATCTC and GGACGAGGACATCCTTGGTA, $2.168 \mathrm{~kb}$ ) and cloned into pUC57 to create pEN-L4-promoter-R1 clones, as described (58). Coding DNA sequences of KOR1 (ATGTACGGAAGAGATCCATG and TCAAGGTTTCCATGGTGCTG) and ESMD1 (ATGCTAGCGAAGAATCGG and GGTGGCAGGAGGTGGTCTC) were amplified from WT cDNA with oligonucleotides specified in parenthesis containing appropriate att sites and recombined with pDONR221 or pDONR-P2R-P3 to obtain pEN-L1-KOR1-L2, pEN-L1ESMD1-L2, and pEN-R2-KOR1-L3. For transcriptional reporters, pEN-L4-JAZ1Op-R1 and pEN-L4ESMD1-R1 were recombined with pEN-L1-NLS-3xVEN-L2 into pEDO097, as described (58). pEN-L4KOR1p-R1 was also recombined with pEN-L1-KOR1-L2 into pEDO097 for complementation analysis. For translational reporters, $\mathrm{pEN}-\mathrm{L} 4-K O R 1 p-\mathrm{R} 1$ or cell-type specific promoters were recombined with pEN-L1-CIT-L2 and pEN-R2-KOR1-L3 into a modified pH7m34gw vector named pFR7m34gw, which harbours seed RFP expression (OLE1p:RFP) instead of $\mathrm{Hg}$ resistance for in planta selection, to generate promoter:CIT-KOR1 constructs. Similarly, to obtain ESMD1p:ESMD1-mT and ESMD1p:ESMD1-CIT, we recombined pEN-L4-ESMD1p-R1, pEN-L1-ESMD1-L2 and pEN-R2-mT-L3 or pEN-R2-CIT-L3 into pFR7m34gw (in both cases, fluorescence signals were undetectable, although the constructs were functionally complementing the mutant phenotype). All constructs were verified by Sanger sequencing, and transgenic plants were generated by floral dip with Agrobacterium tumefaciens strain GV3101. Transformed seeds expressing RFP in $T_{1}, T_{2}$ and $T_{3}$ generations were selected by fluorescence microscopy, and segregation analysis was performed in $>12$ independent $T_{2}$ lines. A minimum of two independent $T_{3}$ transgenic lines were used for each construct to perform experiments and verify reproducibility.

\section{Confocal microscopy}

Confocal laser scanning microscopy was performed on Zeiss LSM 700 or LSM 880 instruments. For live imaging, 5-do vertically-grown seedling roots were mounted in $0.5 x \mathrm{MS}$ with $30 \mu \mathrm{g} / \mathrm{ml} \mathrm{PI}$. As kor1 roots are thick and recalcitrant to PI penetration, kor1 genotypes were fixed in $4 \%$ paraformaldehyde, cleared with ClearSee, and stained with Direct Red 23 (Sigma) as described (59). Excitation/ detection ranges were set as follows: VENUS (VEN) and CITRINE (CIT): 514/ 515-545 nm; mTurquoise (mT): 458/ 460-510 nm; Direct Red 23: 514/ 580-615 nm; Propidium iodide (PI): 561/ 600-700 nm. All images shown within one experiment were taken with identical settings, and by analyzing at least 10 individuals per line. Image processing was performed in Fiji. Z-Stacks were displayed as texture-based volume renderings using the 3DViewer plugin of Fiji. 


\section{Suppressor screen and mapping by NGS sequencing}

Approximately 5000 seeds $(0.1 \mathrm{~g})$ of kor1-4 JGP were mutagenized with ethyl methanesulfonate (EMS, Sigma) as described (6). Resulting $M_{1}$ plants were either harvested individually ( $n=1243$ ) or in pools of 12 ( $n=230) .20 M_{2}$ seedlings were screened from individually harvested plants, and 480 $M_{2} S$ were screened from each pool to enlarge both the screening breadth and depth. A total of 135 $260 \mathrm{M}_{2}$ seedlings, from $4003 \mathrm{M}_{1}$ plants, were assayed for lack of JGP activity in 5-do kor1-4 seedlings by live GUS staining as described (6). To increase the screen stringency and avoid the recovery of false positives, $\mathrm{M}_{2}$ seedlings were shifted from $21^{\circ} \mathrm{C}$ to $26^{\circ} \mathrm{C} 24 \mathrm{~h}$ prior GUS staining as kor1 mutants are known exacerbate their phenotypes at higher $T$ (15). Putative $M_{2}$ suppressors were transferred to soil and crossed to JA-deficient (aos, opr3, jar1) and JA-insensitive (coi1) mutants to avoid the recovery of expected genes, and back crossed to kor1-4 JGP for segregation analysis, phenotype confirmation, and mapping population development. esmd1-3 was identified as a JGP suppressor of kor1-4 by pooling 120 individuals lacking JGP reporter activity from an $\mathrm{BC}_{1} \mathrm{~F}_{2}$ population and sequencing the bulk segregants by whole genome sequencing. Genomic DNA extraction was as in (6). Library preparation (Illumina Shotgun TruSeq DNA PCR-free) and Illumina sequencing on a HiSeq $X$ platform with a 150bp PE read length was performed by Macrogen. The output of $15 \mathrm{~Gb}$ resulted in an average of 118 sequencing depth for each base across the genome. EMS-generated SNPs were identified as described (6), with updated software tools. Sequence reads were mapped to the TAIR10 Arabidopsis thaliana genome with bowtie2 aligner (v. 2.3.1, parameter-end-to-end). Alignment files were converted to BAM with SAMtools ( $v$ 1.8), and SNPs calling was performed with GATK tool (v.4.1.0.0). Common SNPs with the kor1-4 JGP parental line were filtered out with the intersectBed tool from BEDTools utilities (v.2.22.1). The SNPEff tool (v.2.0.4 RC1) was used to predict the effect of the SNPs in coding regions. SNP frequencies (the number of reads supporting a given SNP over the total number of reads covering the SNP location) were extracted using the Unix command awk and plotted with R. Candidate SNPs were identified in genomic regions with high SNP frequencies (0.5-1) linked to the causal SNP, which had the expected frequency of 1 . Validation of the candidate SNP was done by allelism test and by complementation by transformation.

\section{Cell wall composition analysis}

Alcohol-insoluble residue (AIR) was extracted from shoots and roots of 12-do seedlings as previously described (60). Each biological replicate consisted of $\sim 100$ shoots ( $\sim 150 \mathrm{mg} \mathrm{FW}$ ) or $\sim 300$ roots ( $\sim 80$ $\mathrm{mg} \mathrm{FW}$ ). A slurry solution of AIR ( $1 \mathrm{mg} / \mathrm{mL}$ water) was prepared for each sample and homogenized using a ball mill followed by sonication. Matrix polysaccharide composition of $300 \mu \mathrm{g}$ of AIR after 2 $M$ trifluoroacetic acid hydrolysis was analyzed via high-performance anion-exchange 
chromatography with pulsed amperometric detection (HPAEC-PAD), similar to (60), but on a 940 Professional IC Vario ONE/ChS/PP/LPG instrument (Metrohm) equipped with Metrosep Carb2 250/4.0 analytical and guard columns. Each run consisted of neutral sugar separation ( $22 \mathrm{~min} ; 2 \mathrm{mM}$ sodium hydroxide and $3.4 \mathrm{mM}$ sodium acetate isocratic gradient), followed by uronic acid separation (23 min; $100 \mathrm{mM}$ sodium hydroxide and $170 \mathrm{mM}$ sodium acetate), and re-equilibration (14 min; starting eluents) steps.

Cellulose was quantified based on the two-step sulfuric acid hydrolysis method described by (61), with some modifications. Aliquots of AIR (200 $\mathrm{gg}$ each) were first pretreated with concentrated sulfuric acid (to swell cellulose) or were directly used for Seaman hydrolysis (to measure noncrystalline glucose), using ribose as an internal standard. Hydrolysed glucose was quantified using the HPAEC-PAD system described above but with a shorter run: $2 \mathrm{mM}$ sodium hydroxide and 3.4 $\mathrm{mM}$ sodium acetate isocratic gradient ( $22 \mathrm{~min}$ ), followed by a $3 \mathrm{~min}$ rinse with $80 \mathrm{mM}$ sodium hydroxide and $136 \mathrm{mM}$ sodium acetate, and a 4 min re-equilibration with starting eluent.

\section{Sectioning, segmentation and cell analysis}

Roots from vertically-grown 5-do seedlings were vacuum infiltrated and fixed in glutaraldehyde: formaldehyde: $50 \mathrm{mM}$ sodium phosphate buffer $(\mathrm{pH7.2})(2: 5: 43, \mathrm{v} / \mathrm{v} / \mathrm{v})$ for $1 \mathrm{~h}$, dehydrated through an EtOH series, and embedded in Technovit 7100 resin (Heraeus Kulzer) as described (58). Samples were sectioned on a Microm HM355S microtome with a carbide knife (Histoserve) into $5 \mu \mathrm{m}$ sections, mounted in $10 \%$ glycerol, and cell walls were visualized under dark-field of a Zeiss Axiolmager microscope fitted with an AxioCam MRm camera. TIF images were segmented with PlantSeg (62) using preset parameters of the prediction model "lightsheet_unet_bce_dice_ds1x", which empirically segmented our images most accurately. For display purposes only, dark field images were inverted and segmented images re-coloured in Photoshop to visualize different cell types more easily.

\section{Root growth and tropism assays}

Primary root length was evaluated in 7-do seedlings as described (6) and root growth rate was determined by measuring primary root length in 4-do seedlings for 6 consecutive days. Root diameter was assessed in 7-do seedlings by imaging vertically grown seedlings on a Leica M165 FC stereomicroscope fitted with a Leica MC170 HD camera, measuring the root thickness in the early differentiation zone (marked by the appearance of root hairs). Root gravitropism assays were performed on vertically-grown 5-do seedlings by rotating the plates by $90^{\circ}$ and evaluating root 
bending angles $24 \mathrm{~h}$ after the rotation on scanned images with Fiji. For root hydrotropism assays, 5do seedlings were transferred to split-agar plates containing either mock (MS/ MS) or 400 mM mannitol (MS/ mannitol) by aligning root tips $3 \mathrm{~mm}$ from the split-media boundary (63). Root bending angles were evaluated $24 \mathrm{~h}$ after transfer to split-agar plates, by analyzing scanned images with Fiji as described (63).

\section{Statistical analysis}

Box plots, multiple comparisons [analysis of variance (ANOVA) followed by Tukey's honest significant difference (HSD) test], and circular histograms were performed in R.

\section{REFERENCES}

1. G. A. Howe, I. T. Major, A. J. Koo, Modularity in Jasmonate Signaling for Multistress Resilience. Annu Rev Plant Biol 69, 387-415 (2018).

2. J. Browse, J. G. Wallis, Arabidopsis Flowers Unlocked the Mechanism of Jasmonate Signaling. Plants (Basel) 8, (2019).

3. Z. Yang, Y. Huang, J. Yang, S. Yao, K. Zhao, D. Wang, Q. Qin, Z. Bian, Y. Li, Y. Lan, T. Zhou, H. Wang, C. Liu, W. Wang, Y. Qi, Z. Xu, Y. Li, Jasmonate Signaling Enhances RNA Silencing and Antiviral Defense in Rice. Cell Host Microbe 28, 89-103 e108 (2020).

4. A. A. Agrawal, Current trends in the evolutionary ecology of plant defence. Functional Ecology 25, 420-432 (2011).

5. D. Goodspeed, E. W. Chehab, A. Min-Venditti, J. Braam, M. F. Covington, Arabidopsis synchronizes jasmonate-mediated defense with insect circadian behavior. Proc Natl Acad Sci U S A 109, 4674-4677 (2012).

6. I. F. Acosta, D. Gasperini, A. Chetelat, S. Stolz, L. Santuari, E. E. Farmer, Role of NINJA in root jasmonate signaling. Proc Natl Acad Sci U S A 110, 15473-15478 (2013).

7. I. A. Penninckx, B. P. Thomma, A. Buchala, J. P. Metraux, W. F. Broekaert, Concomitant activation of jasmonate and ethylene response pathways is required for induction of a plant defensin gene in Arabidopsis. Plant Cell 10, 2103-2113 (1998).

8. Q. Guo, I. T. Major, G. A. Howe, Resolution of growth-defense conflict: mechanistic insights from jasmonate signaling. Curr Opin Plant Biol 44, 72-81 (2018).

9. M. L. Campos, J. H. Kang, G. A. Howe, Jasmonate-triggered plant immunity. J Chem Ecol 40, 657-675 (2014).

10. C. Wasternack, I. Feussner, The Oxylipin Pathways: Biochemistry and Function. Annu Rev Plant Biol, (2018).

11. S. Mielke, D. Gasperini, Interplay between Plant Cell Walls and Jasmonate Production. Plant Cell Physiol 60, 2629-2637 (2019).

12. D. J. Cosgrove, Plant cell wall extensibility: connecting plant cell growth with cell wall structure, mechanics, and the action of wall-modifying enzymes. J Exp Bot 67, 463476 (2016).

13. L. Denness, J. F. McKenna, C. Segonzac, A. Wormit, P. Madhou, M. Bennett, J. Mansfield, C. Zipfel, T. Hamann, Cell wall damage-induced lignin biosynthesis is 
regulated by a reactive oxygen species- and jasmonic acid-dependent process in Arabidopsis. Plant Physiol 156, 1364-1374 (2011).

14. C. Ellis, I. Karafyllidis, C. Wasternack, J. G. Turner, The Arabidopsis mutant cev1 links cell wall signaling to jasmonate and ethylene responses. Plant Cell 14, 1557-1566 (2002).

15. D. R. Lane, A. Wiedemeier, L. Peng, H. Hofte, S. Vernhettes, T. Desprez, C. H. Hocart, R. J. Birch, T. I. Baskin, J. E. Burn, T. Arioli, A. S. Betzner, R. E. Williamson, Temperature-sensitive alleles of RSW2 link the KORRIGAN endo-1,4-beta-glucanase to cellulose synthesis and cytokinesis in Arabidopsis. Plant Physiol 126, 278-288 (2001).

16. L. Lei, T. Zhang, R. Strasser, C. M. Lee, M. Gonneau, L. Mach, S. Vernhettes, S. H. Kim, J. C. D, S. Li, Y. Gu, The jiaoyao1 Mutant Is an Allele of korrigan1 That Abolishes Endoglucanase Activity and Affects the Organization of Both Cellulose Microfibrils and Microtubules in Arabidopsis. Plant Cell 26, 2601-2616 (2014).

17. J. Lopez-Cruz, I. Finiti, E. Fernandez-Crespo, O. Crespo-Salvador, P. Garcia-Agustin, C. Gonzalez-Bosch, Absence of endo-1,4-beta-glucanase KOR1 alters the jasmonatedependent defence response to Pseudomonas syringae in Arabidopsis. Journal of Plant Physiology 171, 1524-1532 (2014).

18. L. Peng, Y. Kawagoe, P. Hogan, D. Delmer, Sitosterol-beta-glucoside as primer for cellulose synthesis in plants. Science 295, 147-150 (2002).

19. T. Vain, E. F. Crowell, H. Timpano, E. Biot, T. Desprez, N. Mansoori, L. M. Trindade, S. Pagant, S. Robert, H. Hofte, M. Gonneau, S. Vernhettes, The Cellulase KORRIGAN Is Part of the Cellulose Synthase Complex. Plant Physiology 165, 1521-1532 (2014).

20. O. Hamant, E. S. Haswell, Life behind the wall: sensing mechanical cues in plants. BMC Biol 15, 59 (2017).

21. J. K. Polko, J. J. Kieber, The Regulation of Cellulose Biosynthesis in Plants. Plant Cell 31, 282-296 (2019).

22. L. Peng, C. H. Hocart, J. W. Redmond, R. E. Williamson, Fractionation of carbohydrates in Arabidopsis root cell walls shows that three radial swelling loci are specifically involved in cellulose production. Planta 211, 406-414 (2000).

23. A. Chaudhary, X. Chen, J. Gao, B. Lesniewska, R. Hammerl, C. Dawid, K. Schneitz, The Arabidopsis receptor kinase STRUBBELIG regulates the response to cellulose deficiency. PLoS Genet 16, e1008433 (2020).

24. E. E. Farmer, D. Gasperini, I. F. Acosta, The squeeze cell hypothesis for the activation of jasmonate synthesis in response to wounding. New Phytologist 204, 282-288 (2014).

25. T. Engelsdorf, N. Gigli-Bisceglia, M. Veerabagu, J. F. McKenna, L. Vaahtera, F. Augstein, D. Van der Does, C. Zipfel, T. Hamann, The plant cell wall integrity maintenance and immune signaling systems cooperate to control stress responses in Arabidopsis thaliana. Sci Signal 11, (2018).

26. S. Rips, N. Bentley, I. S. Jeong, J. L. Welch, A. von Schaewen, H. Koiwa, Multiple Nglycans cooperate in the subcellular targeting and functioning of Arabidopsis KORRIGAN1. Plant Cell 26, 3792-3808 (2014).

27. P. Marhavy, A. Kurenda, S. Siddique, V. Denervaud Tendon, F. Zhou, J. Holbein, M. S. Hasan, F. M. Grundler, E. E. Farmer, N. Geldner, Single-cell damage elicits regional, nematode-restricting ethylene responses in roots. EMBO J 38, (2019). 
28. M. Li, F. Wang, S. Li, G. Yu, L. Wang, Q. Li, X. Zhu, Z. Li, L. Yuan, P. Liu, Importers drive leaf-to-leaf transmission of jasmonic acid in wound induced systemic immunity. $\mathrm{Mol}$ Plant, (2020).

29. A. Schulze, M. Zimmer, S. Mielke, H. Stellmach, C. W. Melnyk, B. Hause, D. Gasperini, Wound-Induced Shoot-to-Root Relocation of JA-Ile Precursors Coordinates Arabidopsis Growth. Mol Plant 12, 1383-1394 (2019).

30. M. M. Marques-Bueno, A. K. Morao, A. Cayrel, M. P. Platre, M. Barberon, E. Caillieux, V. Colot, Y. Jaillais, F. Roudier, G. Vert, A versatile Multisite Gateway-compatible promoter and transgenic line collection for cell type-specific functional genomics in Arabidopsis. Plant J 85, 320-333 (2016).

31. S. Verger, S. Chabout, E. Gineau, G. Mouille, Cell adhesion in plants is under the control of putative O-fucosyltransferases. Development 143, 2536-2540 (2016).

32. Y. Takenaka, K. Kato, M. Ogawa-Ohnishi, K. Tsuruhama, H. Kajiura, K. Yagyu, A. Takeda, Y. Takeda, T. Kunieda, I. Hara-Nishimura, T. Kuroha, K. Nishitani, Y. Matsubayashi, T. Ishimizu, Pectin RG-I rhamnosyltransferases represent a novel plant-specific glycosyltransferase family. Nat Plants 4, 669-676 (2018).

33. S. Bouton, E. Leboeuf, G. Mouille, M. T. Leydecker, J. Talbotec, F. Granier, M. Lahaye, H. Hofte, H. N. Truong, QUASIMODO1 encodes a putative membrane-bound glycosyltransferase required for normal pectin synthesis and cell adhesion in Arabidopsis. Plant Cell 14, 2577-2590 (2002).

34. J. Du, A. Kirui, S. Huang, L. Wang, W. J. Barnes, S. Kiemle, Y. Zheng, Y. Rui, M. Ruan, S. Qi, S. H. Kim, T. Wang, D. J. Cosgrove, C. T. Anderson, C. Xiao, Mutations in the Pectin Methyltransferase QUASIMODO2 Influence Cellulose Biosynthesis and Wall Integrity in Arabidopsis thaliana. Plant Cell, (2020).

35. G. Mouille, M. C. Ralet, C. Cavelier, C. Eland, D. Effroy, K. Hematy, L. McCartney, H. N. Truong, V. Gaudon, J. F. Thibault, A. Marchant, H. Hofte, Homogalacturonan synthesis in Arabidopsis thaliana requires a Golgi-localized protein with a putative methyltransferase domain. Plant J 50, 605-614 (2007).

36. K. Hematy, P. E. Sado, A. Van Tuinen, S. Rochange, T. Desnos, S. Balzergue, S. Pelletier, J. P. Renou, H. Hofte, A receptor-like kinase mediates the response of Arabidopsis cells to the inhibition of cellulose synthesis. Curr Biol 17, 922-931 (2007).

37. D. L. Yang, J. Yao, C. S. Mei, X. H. Tong, L. J. Zeng, Q. Li, L. T. Xiao, T. P. Sun, J. Li, X. W. Deng, C. M. Lee, M. F. Thomashow, Y. Yang, Z. He, S. Y. He, Plant hormone jasmonate prioritizes defense over growth by interfering with gibberellin signaling cascade. Proc Natl Acad Sci U S A 109, E1192-1200 (2012).

38. D. Dietrich, L. Pang, A. Kobayashi, J. A. Fozard, V. Boudolf, R. Bhosale, R. Antoni, T. Nguyen, S. Hiratsuka, N. Fujii, Y. Miyazawa, T. W. Bae, D. M. Wells, M. R. Owen, L. R. Band, R. J. Dyson, O. E. Jensen, J. R. King, S. R. Tracy, C. J. Sturrock, S. J. Mooney, J. A. Roberts, R. P. Bhalerao, J. R. Dinneny, P. L. Rodriguez, A. Nagatani, Y. Hosokawa, T. I. Baskin, T. P. Pridmore, L. De Veylder, H. Takahashi, M. J. Bennett, Root hydrotropism is controlled via a cortex-specific growth mechanism. Nat Plants 3, 17057 (2017).

39. M. E. Fernandez-Sanchez, S. Barbier, J. Whitehead, G. Bealle, A. Michel, H. LatorreOssa, C. Rey, L. Fouassier, A. Claperon, L. Brulle, E. Girard, N. Servant, T. Rio-Frio, H. Marie, S. Lesieur, C. Housset, J. L. Gennisson, M. Tanter, C. Menager, S. Fre, S. Robine, E. Farge, Mechanical induction of the tumorigenic beta-catenin pathway by tumour growth pressure. Nature 523, 92-95 (2015). 
40. L. Hoermayer, J. C. Montesinos, P. Marhava, E. Benkova, S. Yoshida, J. Friml, Wounding-induced changes in cellular pressure and localized auxin signalling spatially coordinate restorative divisions in roots. Proc Natl Acad Sci U S A 117, 15322-15331 (2020).

41. W. Zhou, J. L. Lozano-Torres, I. Blilou, X. Zhang, Q. Zhai, G. Smant, C. Li, B. Scheres, A Jasmonate Signaling Network Activates Root Stem Cells and Promotes Regeneration. Cell 177, 942-956 e914 (2019).

42. A. Boudaoud, An introduction to the mechanics of morphogenesis for plant biologists. Trends Plant Sci 15, 353-360 (2010).

43. A. Kurenda, C. T. Nguyen, A. Chetelat, S. Stolz, E. E. Farmer, Insect-damaged Arabidopsis moves like wounded Mimosa pudica. Proc Natl Acad Sci U S A 116, 26066-26071 (2019).

44. D. Basu, E. S. Haswell, Plant mechanosensitive ion channels: an ocean of possibilities. Curr Opin Plant Biol 40, 43-48 (2017).

45. A. J. Bidhendi, A. Geitmann, Methods to quantify primary plant cell wall mechanics. J Exp Bot 70, 3615-3648 (2019).

46. L. Michels, V. Gorelova, Y. Harnvanichvech, J. W. Borst, B. Albada, D. Weijers, J. Sprakel, Complete microviscosity maps of living plant cells and tissues with a toolbox of targeting mechanoprobes. Proc Natl Acad Sci U S A 117, 18110-18118 (2020).

47. D. Basu, E. S. Haswell, The Mechanosensitive Ion Channel MSL10 Potentiates Responses to Cell Swelling in Arabidopsis Seedlings. Curr Biol 30, 2716-2728 e2716 (2020).

48. S. E. Murthy, A. E. Dubin, T. Whitwam, S. Jojoa-Cruz, S. M. Cahalan, S. A. R. Mousavi, A. B. Ward, A. Patapoutian, OSCA/TMEM63 are an Evolutionarily Conserved Family of Mechanically Activated Ion Channels. Elife 7, (2018).

49. F. Yuan, H. Yang, Y. Xue, D. Kong, R. Ye, C. Li, J. Zhang, L. Theprungsirikul, T. Shrift, B. Krichilsky, D. M. Johnson, G. B. Swift, Y. He, J. N. Siedow, Z. M. Pei, OSCA1 mediates osmotic-stress-evoked Ca2+ increases vital for osmosensing in Arabidopsis. Nature 514, 367-371 (2014).

50. B. Wachananawat, T. Kuroha, Y. Takenaka, H. Kajiura, S. Naramoto, R. Yokoyama, K. Ishizaki, K. Nishitani, T. Ishimizu, Diversity of Pectin Rhamnogalacturonan I Rhamnosyltransferases in Glycosyltransferase Family 106. Front Plant Sci 11, 997 (2020).

51. F. A. Pettolino, C. Walsh, G. B. Fincher, A. Bacic, Determining the polysaccharide composition of plant cell walls. Nat Protoc 7, 1590-1607 (2012).

52. T. Wang, Y. B. Park, D. J. Cosgrove, M. Hong, Cellulose-Pectin Spatial Contacts Are Inherent to Never-Dried Arabidopsis Primary Cell Walls: Evidence from Solid-State Nuclear Magnetic Resonance. Plant Physiol 168, 871-884 (2015).

53. S. Robinson, A. Burian, E. Couturier, B. Landrein, M. Louveaux, E. D. Neumann, A. Peaucelle, A. Weber, N. Nakayama, Mechanical control of morphogenesis at the shoot apex. J Exp Bot 64, 4729-4744 (2013).

54. M. L. Campos, Y. Yoshida, I. T. Major, D. de Oliveira Ferreira, S. M. Weraduwage, J. E. Froehlich, B. F. Johnson, D. M. Kramer, G. Jander, T. D. Sharkey, G. A. Howe, Rewiring of jasmonate and phytochrome $B$ signalling uncouples plant growth-defense tradeoffs. Nat Commun 7, 12570 (2016). 
55. I. T. Major, Q. Guo, J. Zhai, G. Kapali, D. M. Kramer, G. A. Howe, A Phytochrome BIndependent Pathway Restricts Growth at High Levels of Jasmonate Defense. Plant Physiol 183, 733-749 (2020).

56. J. Ruan, Y. Zhou, M. Zhou, J. Yan, M. Khurshid, W. Weng, J. Cheng, K. Zhang, Jasmonic Acid Signaling Pathway in Plants. Int J Mol Sci 20, (2019).

57. J. M. Kim, T. K. To, A. Matsui, K. Tanoi, N. I. Kobayashi, F. Matsuda, Y. Habu, D. Ogawa, T. Sakamoto, S. Matsunaga, K. Bashir, S. Rasheed, M. Ando, H. Takeda, K. Kawaura, M. Kusano, A. Fukushima, T. A. Endo, T. Kuromori, J. Ishida, T. Morosawa, M. Tanaka, C. Torii, Y. Takebayashi, H. Sakakibara, Y. Ogihara, K. Saito, K. Shinozaki, A. Devoto, M. Seki, Acetate-mediated novel survival strategy against drought in plants. Nat Plants 3, 17097 (2017).

58. D. Gasperini, A. Chetelat, I. F. Acosta, J. Goossens, L. Pauwels, A. Goossens, R. Dreos, E. Alfonso, E. E. Farmer, Multilayered Organization of Jasmonate Signalling in the Regulation of Root Growth. PLoS Genetics 11, e1005300 (2015).

59. R. Ursache, T. G. Andersen, P. Marhavy, N. Geldner, A protocol for combining fluorescent proteins with histological stains for diverse cell wall components. Plant $J$ 93, 399-412 (2018).

60. C. Voiniciuc, M. H. Schmidt, A. Berger, B. Yang, B. Ebert, H. V. Scheller, H. M. North, B. Usadel, M. Gunl, MUCILAGE-RELATED10 Produces Galactoglucomannan That Maintains Pectin and Cellulose Architecture in Arabidopsis Seed Mucilage. Plant Physiol 169, 403-420 (2015).

61. T. H. Yeats, H. Sorek, D. E. Wemmer, C. R. Somerville, Cellulose Deficiency Is Enhanced on Hyper Accumulation of Sucrose by a H+-Coupled Sucrose Symporter. Plant Physiol 171, 110-124 (2016).

62. A. Wolny, L. Cerrone, A. Vijayan, R. Tofanelli, A. V. Barro, M. Louveaux, C. Wenzl, S. Strauss, D. Wilson-Sanchez, R. Lymbouridou, S. S. Steigleder, C. Pape, A. Bailoni, S. Duran-Nebreda, G. W. Bassel, J. U. Lohmann, M. Tsiantis, F. A. Hamprecht, K. Schneitz, A. Maizel, A. Kreshuk, Accurate and versatile 3D segmentation of plant tissues at cellular resolution. Elife 9, (2020).

63. R. Antoni, D. Dietrich, M. J. Bennett, P. L. Rodriguez, Hydrotropism: Analysis of the Root Response to a Moisture Gradient. Methods Mol Biol 1398, 3-9 (2016).

\section{ACKNOWLEDGEMENTS}

We thank Edward E. Farmer for enabling the initiation of this work by gifting the kor1-4 and kor1-5 EMS alleles originally identified in his lab, and for critical comments on the manuscript. We also thank Grégory Mouille for sharing esmd1-1 seeds, Ivan F. Acosta for gifting the pFR7m34gw plasmid, Christine Wagner for assistance with AIR sample preparation, Bo Yang for HPAEC-PAD maintenance, Claus Wasternack and members of the Gasperini lab for critical discussions.

\section{FUNDING}

This work was supported by the Leibniz Institute of Plant Biochemistry from the Leibniz Association, the German Research Foundation (DFG grant GA2419/2-1) to D.G. and (DFG grant 414353267) to C.V., and a Marie Skłodowska-Curie postdoctoral fellowship to M.K.M. 


\section{AUTHOR CONTRIBUTIONS}

S.M. and D.G. designed research; S.M, M.Z., M.K.M. and D.G. performed research; S.M., M.K.M., R.D., and D.G. analyzed data; H.S. and B.H. quantified JA-lle levels; C.V. performed cell wall composition analyses; S.M. and D.G. wrote the manuscript with input from all authors.

\section{COMPETING INTERESTS}

The authors declare no competing interests.

\section{FIGURES and TABLES}

Figures 1 to 6.

\section{SUPPLEMENTARY MATERIALS}

Supplementary Figures S1 to S7. 